\title{
Mechanoresponsive Materials for Drug Delivery: Harnessing Forces for Controlled Release
}

\author{
Julia Wang ${ }^{\mathrm{a}}$, Jonah A. Kaplan ${ }^{\mathrm{a}}$, Yolonda L. Colson ${ }^{\mathrm{b}}$, and Mark W. Grinstaff ${ }^{\mathrm{a} \star}$ \\ ${ }^{a}$ Departments of Biomedical Engineering, Chemistry and Medicine, Boston University, \\ 590 Commonwealth Avenue, Boston, MA 02215 \\ 'Division of Thoracic Surgery, Department of Surgery, Brigham and Women's Hospital, \\ Boston, MA 02115
}

Abstract:

Mechanically-activated delivery systems harness existing physiological and/or externally-applied forces to provide spatiotemporal control over the release of active agents. Current strategies to deliver therapeutic proteins and drugs use three types of mechanical stimuli: compression, tension, and shear. Based on the intended application, each stimulus requires specific material selection, in terms of substrate composition and size (e.g., macrostructured materials and nanomaterials), for optimal in vitro and in vivo performance. For example, compressive systems typically utilize hydrogels or elastomeric substrates that respond to and withstand cyclic compressive loading, whereas, tension-responsive systems use composites to compartmentalize payloads. Finally, shear-activated systems are based on nanoassemblies or microaggregates that respond to physiological or externally-applied shear stresses. In order to provide a comprehensive assessment of current research on mechanoresponsive drug delivery, the mechanical stimuli intrinsically present in the human body are first discussed, along with the mechanical forces typically applied during medical device interventions, followed by in-depth descriptions of compression, tension, and shear-mediated drug delivery devices. We conclude by summarizing the progress of current research aimed at integrating mechanoresponsive elements within these devices, identifying additional clinical opportunities for mechanically-activated systems, and discussing future prospects.

Table of Contents:

1. Introduction

2. Compression-responsive systems

2.1. Elastomeric deformation

2.2. Hydrogel deformation

3. Tension-responsive systems

3.1. Hybrid composites with capsular/ particulate species

3.2. Layered composites

3.2.1 Polyelectrolyte films

3.2.2 Wetting of superhydrophobic systems

4. Shear-responsive systems

4.1. Liposome deformation

4.2. Particle aggregation and dispersion

4.3. Supramolecular disassembly

5. Conclusions and future perspectives 


\section{Introduction}

The delivery of therapeutic agents to a specific location with optimal dose and duration remains a significant clinical challenge. This multifaceted problem is being investigated using a myriad of drug delivery strategies because systemic drug administration-although widely used in the clinic-typically requires multiple doses to treat diseased tissue. However, this leads to significant and widespread off-target side effects due to exposure of healthy tissue. Stimuli-responsive materials are well-suited for applications in drug delivery, actively releasing their drug payloads in response to either physiological or externally-applied triggers. This spatiotemporal control over drug release is widely demonstrated for stimuli such as: $\mathrm{pH}$ [1-11], temperature $[1,9,10,12-$ 21], light [22-26], ionic strength [27-29], electrical potential [30-37], and applied magnetic fields [38-48]. While some of these systems ultimately undergo a mechanical change, such as deformation, swelling, or change in modulus (i.e., when temperatures reach above the lower critical solution temperature or below the upper critical solution temperature), they will not be discussed as these systems are previously reviewed. Instead, this review highlights recent exciting breakthroughs with stimuli-responsive systems that respond directly to mechanical forces and summarizes pioneering reports that have launched the field.

Mechanically-activated systems are triggered by mechanical forces in the body that either occur physiologically or are exerted on the body by external devices, both over a wide magnitude (Figure 1). Generally, an unopposed force exerted on an object accelerates its motion. The distribution of the force on the object is described as the mechanical stress, which can result in deformation. Microscopic cellular forces [49-54] are present and coordinate into macroscopic forces for processes such as wound repair and inflammation. Further coordination results in the exertion of even greater forces by various systems, such as the musculoskeletal [55,56], cardiovascular [57-59], and respiratory systems [60,61]. Alternatively, external triggers are applied by medical devices such as stents [62-65] and catheters [66,67] that mechanically open blocked or narrowed structures, or are applied by another user or self-exerted to control administration. Therefore, drug and protein delivery systems that respond to mechanical forces serve as innovative solutions to control on-demand release within a physiological

$\begin{array}{llllll}\text { Force Exerted: } \quad 10^{-9} \mathrm{~N} & 10^{-6} \mathrm{~N} & 10^{-3} \mathrm{~N} & 1 \mathrm{~N} & 10^{3} \mathrm{~N}\end{array}$

Physiological

\section{Cellular Forces}

\section{Cardiovascular, Musculoskeletal}

External

\section{Stents}

Catheters

User

Figure 1. Physiological and external forces and their relative magnitudes present in the body. 
environment. Designing such mechanoresponsive systems that account for the dynamic nature of the human body will bring about novel solutions to clinical challenges.

Mechanical stimuli are quantified by force and displacement (Figure 2). In compression, a force is applied, resulting in an equal but opposing force along the same axis, generally reducing the object's length along that direction. Similarly, an object under tension is pulled or stretched, lengthening the object along the axis. This force, and resulting deformation, can be converted into stress and strain. For engineering stress $(\sigma)$, the force is normalized by the cross sectional area while engineering strain $(\varepsilon)$ calculates the relative change in displacement - the difference in length divided by the original length. Instead of applying forces normal to the cross section, shear forces are applied parallel to the object's cross section. Shear stress is similarly defined as the parallel force divided by the cross sectional area acted upon; shear strain is the strain in the parallel direction. The overall elastic material property is expressed by Young's modulus: $E=$ stress/ strain. The shear modulus is defined as $G=E /(2(1+v))$, where $v$ is Poisson's ratio, which describes the expansion of the material along the axis compared to the compression perpendicular to the axis.

While there are relatively few reports of mechanoresponsive drug delivery systems [68], they cover the breadth of mechanical forces: compression, tension, and shear.

A. Compression

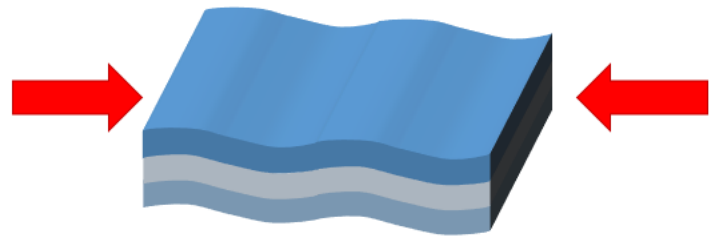

B. Tension

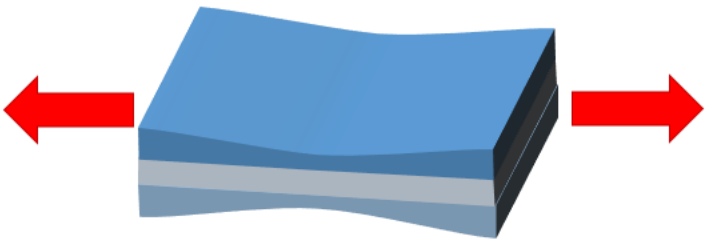

C. Shear

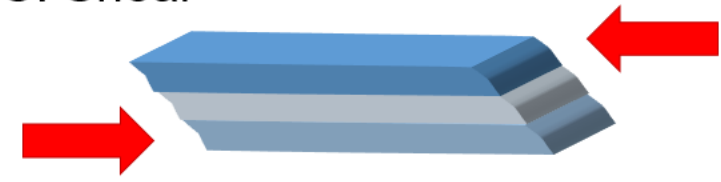

Figure 2. Schematic representation of compressive, tensile, and shear forces. Mechanoresponsive drug delivery is attractive due to the ease of applying compressive, tensile, and shear stimuli, and to the ubiquity of these forces in the human body. While ultrasound is also considered a mechanical stimulus, several recent reviews have been published on ultrasoundtriggered drug delivery [69-83], and thus will not be discussed here. The scope of the current review focuses on drug delivery systems that utilize compression, tension, and shear, and are categorized according to the respective forces used for mechanical stimulation of drug release. 


\section{Compression-responsive systems}

Compressive delivery systems require substrates that respond to and withstand compressive loading. Commonly used materials for compression are elastomeric substrates. Elastomers are viscoelastic polymers - that is, they have viscous (resistance to flow) and elastic properties (tendency to return to its original shape after removal of stress) with time-dependent strain rate. Examples of elastomers in biomedical research include rubbers and silicones. As 3D-crosslinked polymer networks, hydrogels also withstand high compressive forces, and thus act as effective compressive systems. Examples of natural hydrogel polymers include alginate, chitosan, collagen, and hyaluronic acid, whereas examples of synthetic hydrogel polymers include poly(hydroxyethylmethacrylate), polyacrylamides, poly(ethylene glycol), poly(vinyl alcohol), and poly( $\mathrm{N}$-isopropyl acrylamide). Due to their biocompatibility and aqueous loading environment, hydrogels are widely used in the clinic and in biomedicine for tissue engineering [84-89], diagnostic [90-92], and drug and protein delivery [93-98] applications. The availability of both types of substrates and the ability to apply compressive forces externally have led to numerous studies on controlling the release of drugs and proteins.

\subsection{Elastomeric deformation}

Elastomeric substrates possess the structural integrity required for compressive release. PY Wang reports one of the earliest successes of a user-controlled compressive system, where a two-compartment silicone implant releases insulin to reduce hyperglycemia in an in vivo diabetic rat model [99]. The first compartment allows the influx of serous fluid to solubilize the insulin powder contained in the second compartment. Compression then drives the efflux of the insulin-dissolved serum. The author demonstrates efficacious insulin delivery from the implant in vivo using diabetic Wistar rats undergoing compressions ( 2 seconds followed by 1 minute massage) once a day, once every 2 days (Figure 3a), once every 3 days, and once every week, with subsequent reduction in blood sugar levels up to $28,44,72$, and 140 days, respectively. Although the insulin 'dose' is similar in each case (i.e., reduction of blood glucose levels last for $\sim 1$ day), the depletion rate increases with longer durations between administration; compressions once a day are effective over 28 stimulation events, while the device is only active for 20 stimulation events when compressed once a week. While the author addresses the retention of insulin bioactivity with this in vivo model, another concern is the variability of forces used to compress the implants; better characterization of the forces exerted is necessary to control consistent delivery.

Yang et al describe another example of cyclical compressive release, demonstrating controlled release of bovine serum albumin (BSA) from porous matrices [100]. In their study, BSA-loaded microspheres are incorporated within block copolymer poly(ethylene glycol)-b-poly(L-lactide) (PELA) scaffolds. Compression of the scaffolds $(1 \mathrm{~Hz})$ for 3 hours each day (4-5\% compressive strain) for 30 days reveal accelerated BSA release from cyclic loading compared to the scaffold under static conditions. Half of the total BSA concentration releases after 4 days under cyclic loading while the release extends to 10 days under static conditions. This result is an improvement over BSA release from PELA microspheres without scaffolds, which release $50 \%$ of their payload after 2 days. The authors note areas for design improvement in their system including reducing the high degree of burst release from both static and compressed samples. 
In contrast to systems solely undergoing compression, Kim et al recently report a microfabricated polydimethylsiloxane

(PDMS) system, containing a microchannel adjacent to a refillable spherical reservoir [101], that is subjected to bending, which induces a range of compressive and tensile stresses and stress magnitudes. When the strain applied to the reservoir exceeds the critical strain $\left(\varepsilon_{c}\right)$, or the strain at which the volume of the deformed reservoir exceeds the volume of the microchannel, the system releases compound due to strain-induced flow and diffusion (Figure $3 b$ ). Using rhodamine $B$ as a model hydrophilic drug, the authors demonstrate control over delivery kinetics, depending on the relative microchannel volume fraction $\left(f_{c h}=\right.$ $\left.V_{c h} / V_{R} \times 100 \%\right)$ and bending radii ( $r=$ 19, 27, $45 \mathrm{~mm}$ ). Without microchannels $\left(f_{c h}=0 \%\right)$, less inward bending (larger bending radius, $r=45 \mathrm{~mm}$, Figure $3 \mathrm{c}$ ) results in more on/off release profile and less release at each bending event ( 2\%), while more inward bending (smaller bending radius, $r=19 \mathrm{~mm}$, Figure $3 d$ ) results in slow continuous diffusion in the absence of a bending trigger, with greater release at each bending event ( $4 \%)$. Longer channels (up to $f_{c h}=9 \%$ ) retain more buffer volume and therefore less rhodamine $B$ releases with each bending event (for $r=19 \mathrm{~mm},<0.5 \%$ releases at $f_{c h}=9 \%$ vs $4 \%$ release at $f_{c h}=0 \%$ ) (Figure 3e). However, the maximal drug release is $\approx 70 \%$, most likely attributed to the dead volume of the system, which the authors suggest can be solved by refilling the system with a syringe.

Alternatively, Larsen et al use a mechanochemical approach to
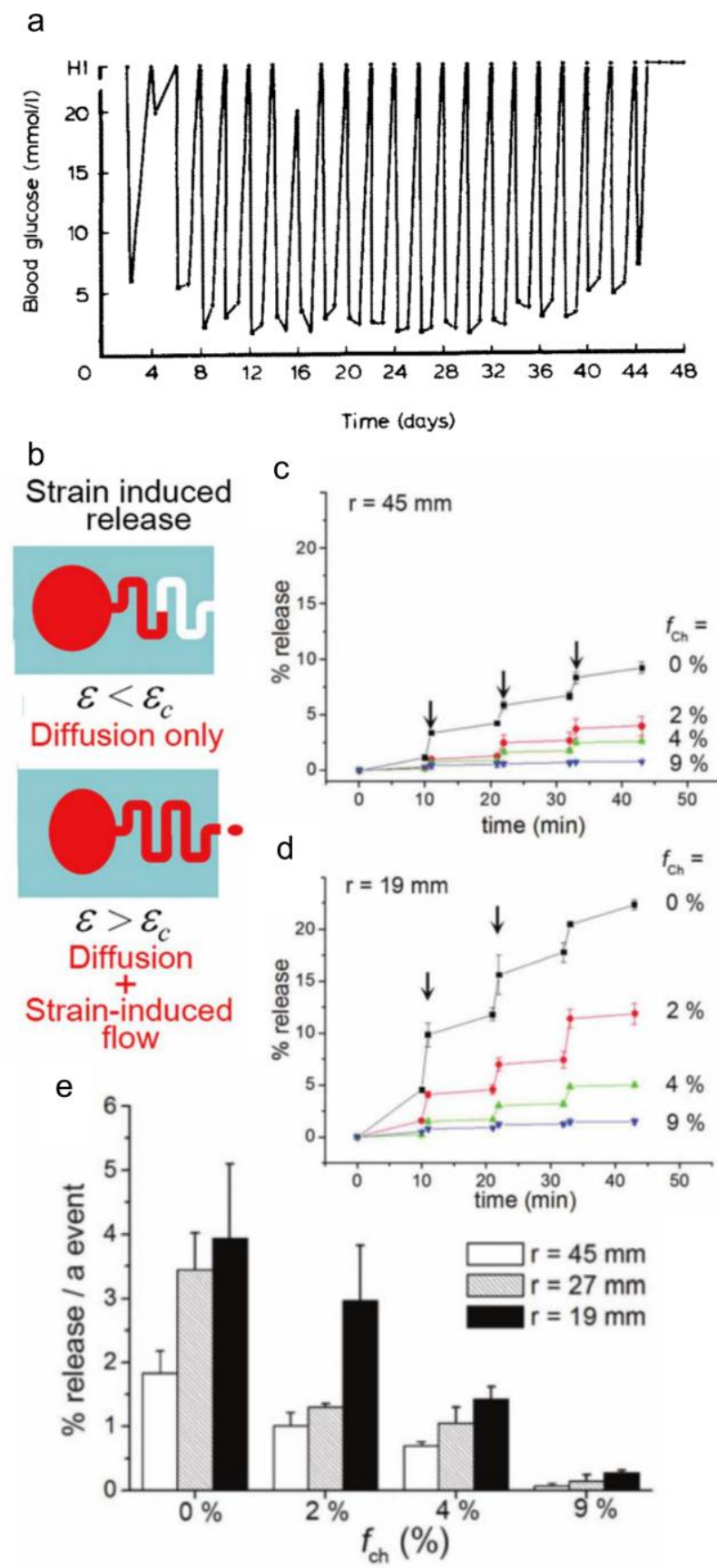

Figure 3. Compressive elastomeric systems. (a) Reduction of blood glucose in rats with the release of insulin from silicone implants upon stimulation every 2 days. (b) Schematic representation of release when compression of the device reaches the critical strain $\left(\varepsilon_{c}\right)$. Release profile of rhodamine $B$ with three bending events with bending radius of (c) $45 \mathrm{~mm}$, and (d) $19 \mathrm{~mm}$. (d) Modulation of release with channel volume. Figures are reprinted with permission from Refs. [99] and [101]. 
activate the release of a furan-derivative through compression [102]. The polyurethanederived network, crosslinked with oxanorbornadiene-based mechanophores, undergoes orthogonal bonds breakage with mechanical compression via a retro-[4+2] cycloaddition reaction, resulting in the formation of an alkyl bond and release of the small molecule, benzyl furfuryl ether. The control system, consisting of physically but not chemicallyincorporated mechanophores, has a baseline release of $\sim 2 \%$, regardless of the compressive pressure applied. In contrast, the mechanosensitive polyurethane network, containing the chemically incorporated mechanophores, shows increasing release from $1 \%$ to $6 \%$ with greater compression (up to $176 \mathrm{MPa}$ ). The authors also demonstrate compression-triggered release of benzyl furfuryl ether with up to 9 consecutive cycles at 35 and $88 \mathrm{MPa}$ for 1 minute (max $\sim 7 \%$ activation). This mechanically-induced bond scission offers a chemical approach to release but limits the release to substrates containing furan functionalities with low release of the incorporated substrate. 


\subsection{Hydrogel deformation}

Lee et al report one of the earliest hydrogel compressive systems, composed of calcium crosslinked alginate, as a device to stimulate neovascularization through the delivery of physically entrapped vascular endothelial growth factor (VEGF) [103,104]. Compression (six cycles with compression for 2 mins and relaxation for 8 mins between cycles) at $25 \%$ or $10 \%$ strain expedites the release rate of VEGF five-fold from the alginate hydrogel or two-fold, respectively, compared to the control ( $0 \%$ strain) (Figure 4a). The authors also demonstrate the in vivo efficacy of these VEGF implants, showing nearly 7-fold greater tissue granulation and more than two-fold increase in blood vessel number in severe combined immunodeficient mice after 7 days of daily mechanical stimulation (three cycles with compression for 1 min followed by 1 min relaxation between cycles at $50 \%$ strain for 1 week), compared to unloaded hydrogels with and without mechanical stimulation. Similarly, in a non-obese diabetic mouse model, blood vessel density increases by 3-fold with VEGF hydrogels under mechanical stimulation (six cycles with compression for 30 seconds, followed by relaxation for 90 seconds, over 1 week) compared to unloaded hydrogels with and without mechanical stimulation (Figures $4 \mathrm{~b}$ and $4 \mathrm{c}$ ). However, in the absence of compression, there is a non-zero release rate reported in vitro $(\sim 2 \mathrm{ng} / \mathrm{min})$ and a significantly higher amount of granulation and blood vessel number (or density) with non-mechanically stimulated VEGF-loaded hydrogels (Figure 4b) compared to unloaded hydrogels. However, this passive release still results in significantly lower granulation and blood vessel density than mechanically-stimulated VEGF-loaded hydrogels in both in vivo models.

Extending the promising nature of alginate hydrogels for the delivery of hydrophobic compounds, $\beta$-cyclodextrin moieties increase drug loading and prolong release through host-guest interactions. $\beta$-cyclodextrin is a 7-membered sugar ring molecule with a hydrophobic center and hydrophilic sides. This, along with 21 hydroxyl groups available for modification, allows higher incorporation and better retention of hydrophobic drugs through van der Waals and hydrophobic complexes [105]. Izawa et al release ondansetron, a hydrophobic anti-emetic drug, from a compressive system of crosslinkable $\beta$-cyclodextrin grafted to alginate [106]. Incorporation of the $\beta$-cyclodextrin moieties increase ondansetron binding to $170 \mathrm{M}^{-1}$, compared to $25 \mathrm{M}^{-1}$ for calciumcrosslinked alginate gels. $\beta$-cyclodextrin-crosslinked alginate hydrogels are mechanically-responsive to compressive strain $(50 \%, 30 \%, 0 \%)$ compared to $\beta$ cyclodextrin-grafted hydrogels (single link to alginate) (Figure 4d), confirming the deformation of the $\beta$-cyclodextrin moiety, through its multi-connectivity with the alginate matrix, is required for mechanoresponsive release rather than enhanced diffusion by water exudation. 1-cycle compressions and 5-cycle compressions at $50 \%$ or $30 \%$ strains ( 5 mins compression followed by 5 mins relaxation) increases release over a 70 hour period (Figure 4e). The deformation due to compressive stimulus decreases ondansetron affinity to $\beta$-cyclodextrin by destabilizing the inclusion complex (50\% strain decreases binding constant to $100 \mathrm{M}^{-1}$ vs $170 \mathrm{M}^{-1}$ with $0 \%$ strain). However, even with the increased affinity of ondansetron, the system still passively releases half of the ondansetron over 18 hours with no mechanical stimulus (vs 7 hours at 50\% strain, Figure 4e).

Similarly, Tan et al report the use of $\beta$-cyclodextrin-conjugate alginate hydrogels, loaded with hydrocortisone acetate, an anti-inflammatory drug, as a potential wound healing implant [107]. Calcium-crosslinked alginate gels (without mono-6-deoxy-6- 
ethylenediamine- $\beta$-cyclodextrin ( $\beta$-CD-EDA) grafts) are not mechanically responsive to $3 \mathrm{kPa}$ compressive stress-that is, mechanically stimulated and non-stimulated hydrogels release equally fast ( $50 \%$ release over $\sim 5$ hours). However, with increasing incorporation of $\beta$-CD-EDA (weight ratio from $50 / 0$ to $27 / 23$ alginate/ $\beta$-CD-EDA), mechanical sensitivity increases and release is prolonged as non-stimulated hydrogels release $50 \%$ over $30+$ hours while stimulated hydrogels release in $\sim 25$ hours. In vitro studies in lipopolysaccharide-activated RAW264.7 murine macrophages demonstrate a nearly 8-fold reduction of nitric oxide production over 72 hours, confirming the antiinflammatory function of the hydrocortisone acetate from mechanically-stimulated hydrogels. However, the mechanical properties of the hydrogel need to be further optimized as there is a tradeoff with increasing $\beta$-CD-EDA concentration, which results

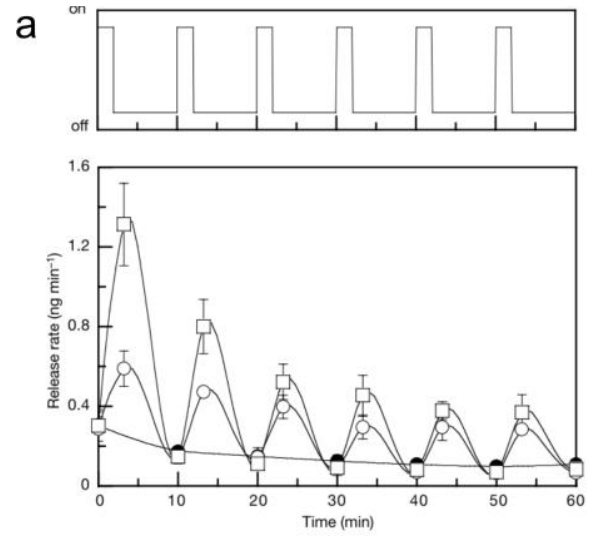

d

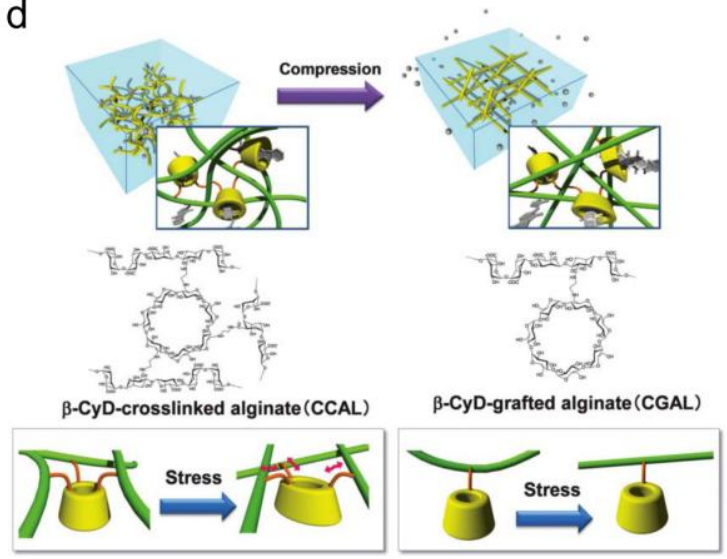

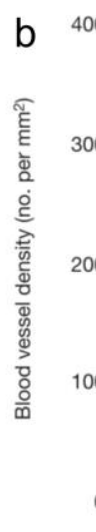

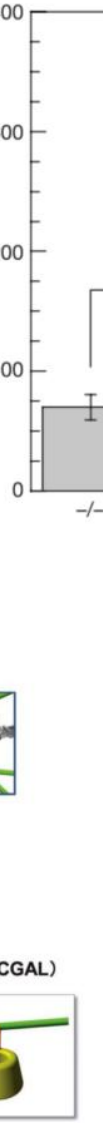

C

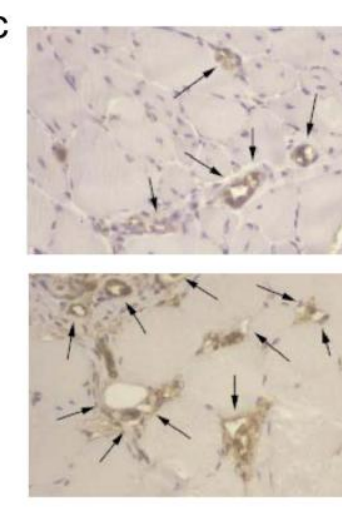

VEGF/stimulation

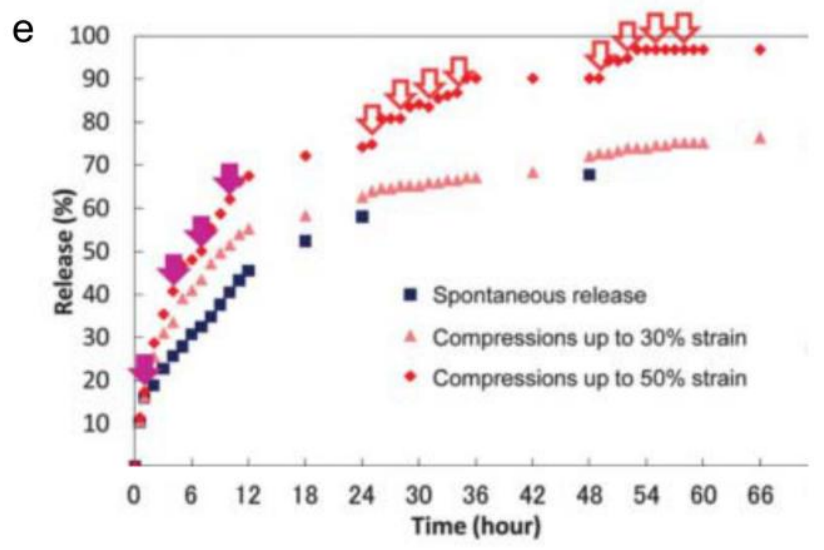

Figure 4. Compressive alginate systems. (a) Release of VEGF in vitro from alginate hydrogels upon mechanical compression. (b) Increase in blood vessel density with loaded and mechanically stimulated hydrogels $(+/+)$, compared to unloaded hydrogels with no mechanical stimulation (-/-), unloaed hydrogels with mechanical stimulation $(-/+)$, and loaded hydrogels with no mechanical stimulation (+/-). (c) Photomicrographs of tissue sections with blood vessels (arrows) in loaded hydrogels without mechanical stimulation (+/-, top) and loaded hydrogels with mechanical stimulation $(+/+$, bottom). (d) Schematic representation of $\beta$-cyclodextrin conjugated alginate system with multiple crosslinks (left) and single link (right). (e) Release profile from crosslinked $\beta$-cyclodextrin alginate gels, modulating release based on strain $(50 \%, 30 \%, 0 \%)$. Solid purple arrows represent one-time compressions, red empty arrows represent five-cycle compressions. Figures are reprinted with permission from Refs. [103] and [106]. 
in decreased overall mechanical strength of the system. For this reason, only one magnitude of compressive stress $(3 \mathrm{kPa}$ ) is described (lower magnitudes would not be indicative of user-controlled compression). Moreover, the leakiness amongst all $\beta-C D-$ EDA and alginate systems is a concern due to undesired drug release, similar to the previous two alginate systems. Thus, opportunities exist to improve mechanical properties and release mechanics as a future user-controlled implant.

Alternative compression-responsive systems composed of materials other than alginate are also reported. Xiao et al use a hyaluronic acid (HA)-based hydrogel containing covalently integrated soft and deformable dexamethasone (DEX) micelles for pain management [108]. The compressive release of anti-inflammatory drug DEX from hydrogel implants would relieve pain for osteoarthritic patients when degraded cartilage undergoes compression through daily activity. The hydrogel system consists of block copolymer micelles (BCM), composed of poly(acrylic acid-graft-2-hydroxyethyl acrylate)block-poly ( $n$-butyl acrylate) tethered to glycidyl methacrylate-modified HA using photoinitiated free-radical polymerization. Owing to its lipophilicity, DEX sequesters within the hydrophobic BCM cores, yet releases in an on-demand fashion through the application

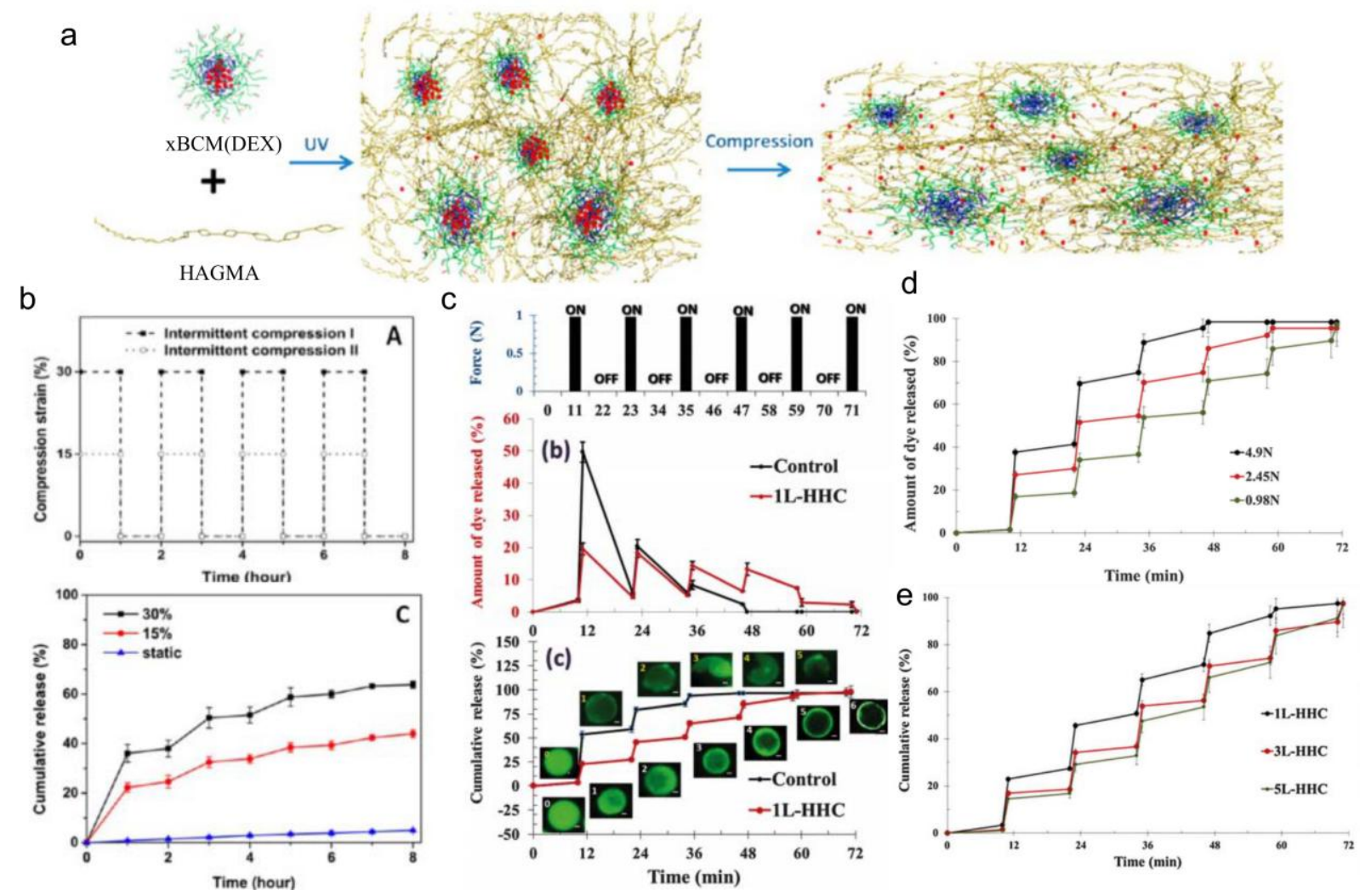

Figure 5. Compressive hydrogel systems. (a) Schematic representation of dexamethasoneencapsulated micelles (xBCM(DEX)) crosslinked to hyaluronic acid-based matrix (HAGMA). (b) Mechanical stimulation (top) and resulting release profile (bottom) from $\times B C M(D E X)$ crosslinked to HAGMA. (c) Mechanical stimulation (top) and corresponding release (middle) and cumulative release (bottom) of FITC encapsulated in chitosan-alginate layers with silica nanoparticles (1L-HHC) compared to chitosan-alginate layers (control). Release is modulated by force applied (d) and addition of more layers (e). Figures are reprinted with permission from Refs. [108] and [109] 
of compression (Figure 5a). With an hour of $30 \%$ compressive strain (12 cycles/hour), DEX releases at $345 \mu \mathrm{g} / \mathrm{hour}$ compared to $6 \mu \mathrm{g} /$ hour under static conditions (Figure $5 \mathrm{~b}$ ); at $15 \%$ compressive strain (12 cycles/hour), DEX also releases but at a rate $1.6 x$ slower compared to $30 \%$ strain (Figure $5 \mathrm{~b}$ ). However, a limitation of the system is the substantial burst release which requires a 3-hour washout period prior to performing experiments. Lastly, release of DEX from non-mechanically-stimulated hydrogels significantly reduces levels of secreted tumor necrosis factor a (and thus reduces RAW264.7 murine macrophage activation) after stimulation by lipopolysaccharide, demonstrating in vitro reduction of inflammation.

Recently, Rajamanickam et al release model drugs from elastic layer-by-layer microparticles $(\sim 17 \mu \mathrm{m})$ via cyclic compression [109]. The alternating layers consist of chitosan (Chi), alginate (Alg), or colloidal silica nanoparticles $\left(\mathrm{SO}_{2}\right)$ : (Chi-Alg-Chi)-(Alg-

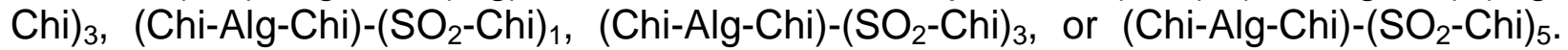
The chitosan layers are covalently crosslinked by glutaraldehyde at freezing temperatures to impart elasticity, while the colloidal silica nanoparticles reinforce the strength of the layers. Mechanical tests via capillary compression, capillary micromechanics, and osmotic pressure demonstrate that (Chi-Alg-Chi)- $\left(\mathrm{SO}_{2}-\mathrm{Chi}\right)_{3}$ deform by more than $98 \%$ under compression with recovery, the shear modulus of the particles increases with greater deposition of $\mathrm{SO}_{2}$-Chi layers, and the critical osmotic pressure also increases with greater deposition of $\mathrm{SO}_{2}$-Chi layers. The microparticle assembly is capable of $30-40 \%$ diffusive loading of small and neutral dyes, such as fluorescein isothiocyanate (FITC) or FITC-dextran $(4 \mathrm{kDa})$, but results in limited loading of polymeric and charged dyes. Nevertheless, FITC-dextran releases from a monolayer of (Chi-Alg-Chi)-( $\mathrm{SO}_{2}$-Chi) $)_{1}$ particles with 4 cyclic compression $(0.98 \mathrm{~N}$ force for 6 seconds, 12 min relaxation). With $\sim 20 \%$ of the dye release at each cycle, the system with silica nanoparticles ((Chi-Alg-Chi)-( $\left.\left(\mathrm{SO}_{2}-\mathrm{Chi}\right)_{5}\right)$ exhibits less burst release compared to the control ((Chi-Alg-Chi)-(Alg-Chi) $\left.)_{3}\right)$ (Figure 5c). Additionally, increasing the magnitude of force applied $(4.9 \mathrm{~N}, 2.45 \mathrm{~N}, 0.98 \mathrm{~N}$, Figure $5 \mathrm{~d})$ results in faster release while increasing the silica-chitosan layers (from (Chi-Alg-Chi)- $\left(\mathrm{SO}_{2}-\mathrm{Chi}\right)_{1}$ to (Chi-Alg$\mathrm{Chi})-\left(\mathrm{SO}_{2}-\mathrm{Chi}\right)_{3}$ and (Chi-Alg-Chi)-( $\left.\left.\mathrm{SO}_{2}-\mathrm{Chi}\right)_{5}\right)$ decreases the release with each cycle $(\sim 15-20 \%$ dye released at each cycle) (Figure 5e). However, the authors do not demonstrate the activity of the released agents as the studies are conducted with model compounds. Furthermore, how the particles would be compressed in vivo is not discussed as the experiments rely on a monolayer of immobilized particles. 


\section{Tension-responsive systems}

Tension-responsive systems are an active area of study in the fields of sensors [110-112], electronics [113-116], and more recently drug delivery. Each of these systems utilizes 'soft' and often elastomeric materials. In drug and protein delivery, tension is an ideal stimulus because of the ubiquity of tension in the dynamic nature of the human body and the increasing use of tension-driven medical devices (i.e., stents, catheters) [117].

While most hydrogels are capable of compressive loading, hydrogels often yield at low tensile strains (i.e., $<50 \%$ strain, [118]). From a highly stretchable interpenetrating alginate-polyacrylamide hydrogel, first developed by Sun et al [119], Zhang et al release horseradish peroxidase and Candida antarctica lipase B by stretching [120]. Biaxial stretching increases the surface area of the hydrogel, allowing faster diffusion of substrate into the enzyme-embedded hydrogel; an increase in surface area linearly correlates with activity of both enzymes. Heterogeneous composite systems, discussed in the following sections, utilize a similar approach, controlling release rates by increasing the surface area exposure and also addressing the inherent leakiness present in homogeneous systems, such as this study, in the absence of the tensile strain trigger.

\subsection{Hybrid composites with capsular/ particulate species}

In contrast to single component systems, heterogeneous composite systems increase drug encapsulation, slow passive drug diffusion, and allow control over bulk material mechanics. The capsular component (micelle, microcapsules, or nanoparticles) of the device provides higher drug encapsulation and acts as an additional barrier to slow the diffusion of drug, especially in the absence of a tension trigger. Furthermore, the composite system separates the bulk mechanics from agent encapsulation, freeing the available choices for substrates.

For example, Hyun et al [121] report a tension-responsive drug delivery system with microcapsule arrays supported on an elastomeric substrate. These isotropic buckled polystyrene (PS) films, processed through thermal cycling, are loaded with model drugs, fluorescein-isothiocyanate (FITC)-labeled dextran or rhodamine B. The final drug delivery device consists of sealed microcapsules, achieved by affixing the buckled PS film to a soft poly(dimethylsiloxane) (PDMS) substrate (Figure 6a). In agreement with theoretical models, increasing strain decreases the height of the microcapsules and increases their spacing, up to $8.5 \%$ strain, at which point the PS film fractures. Repeated stretching of these devices $(0.1 \%$ strain/ second) to various strain magnitudes controls the amount of rhodamine $\mathrm{B}$ release. Higher strain magnitudes $(7.5 \%)$ result in greater rhodamine $B$ released at each event $(8-12 \%)$ while lower strain magnitudes $(1 \%)$ result in less compound released at each event ( 1\%) (Figure 6b). However, when FITC-dextran is loaded into the devices, it does not release under either static or dynamic conditions, which the authors attribute to its macromolecular nature: the PS membrane severely restricts diffusion of macromolecules, such as FITC-dextran, but allows strain-dependent diffusion of small molecules, such as rhodamine B. This system is, therefore, limited to small molecule drug delivery, and thus opportunities exist for mechanoresponsive drug delivery systems to deliver both small and macromolecular therapies and at higher strains.

Xiao et al [122] employ elastomeric hydrogels for the mechanoresponsive release of pyrene, a hydrophobic, fluorescent model drug. Block copolymer micelles (BCM) consist 
of two components: poly( $n$-butyl acrylate) serving as the hydrophobic portion for pyrene loading, and poly(acrylic acid) modified with 2-hydroxyethyl acrylate serving as the hydrophilic portion, which also crosslinks to the bulk polyacrylamide hydrogel by free radical polymerization. Stretched hydrogels transfer their macroscopic deflection to the BCMs through these crosslinks, causing a morphological change in the micelles that favors pyrene release (Figure 6c). Hydrogels stretched to $60 \%$ strain release pyrene at a $2.5 \mathrm{x}$ faster rate over the first extension/relaxation interval than hydrogels stretched to $30 \%$ strain, and release pyrene at a $5 x$ faster rate than hydrogels incubated under static conditions ( $0 \%$ strain) (Figure $6 \mathrm{~d}$, top). In addition, cumulative release of pyrene over 60 minutes (60 cycles over 5 minutes followed by 5 minutes of rest) is greatest for hydrogels stretched to $60 \%$ ( $25 \%$ release), compared to those stretched to $30 \%$ $(\sim 15 \%$ release) and static samples $(\sim 10 \%$ release) (Figure $6 \mathrm{~d}$, bottom). Although the BCM-crosslinked hydrogels withstand up to $350 \%$ strain without failure, it is uncertain how these hydrogels perform at higher strains as the release at $60 \%$ strain is the maximum value reported.

Di et al [123] use a similar approach, embedding alginate microparticles with drug or protein-loaded PLGA nanoparticles into an elastomeric base substrate (Dragon Skin 30) (Figure 6e). The surface area of the alginate depot increases with increasing strain, thus facilitating greater drug diffusion and release. Doxorubicin-formulated devices demonstrate strain-dependent release $(100 \%$ strain releases $\sim 3 x$ more than $0 \%$ strain, with 10 cycles with 2 seconds/ cycle) with cycle-dependent (50\% strain from 0 to 10 cycles) decrease in HeLa viability in vitro with 2D and 3D cultures. Microneedles, composed of hyaluronic acid crosslinked microparticles loaded with insulin, are applied in vivo to the skin of streptozotocin-induced diabetic mice. Blood glucose levels decrease over three 4-hour stretching intervals (10 cycles at $50 \%$ strain), while nonstimulated microneedles initially burst release (still hyperglycemic amounts) and bolus insulin injections reduce blood glucose levels over one 4-hour interval (Figure 6f). Releasing a variety of compounds upon physiological stimulation demonstrates the potential versatility of the system in the clinic. However, control over passive release is necessary to minimize resistance to chemotherapeutics or antibiotics, and to preserve the amount of drug or protein available for release at a later time. 

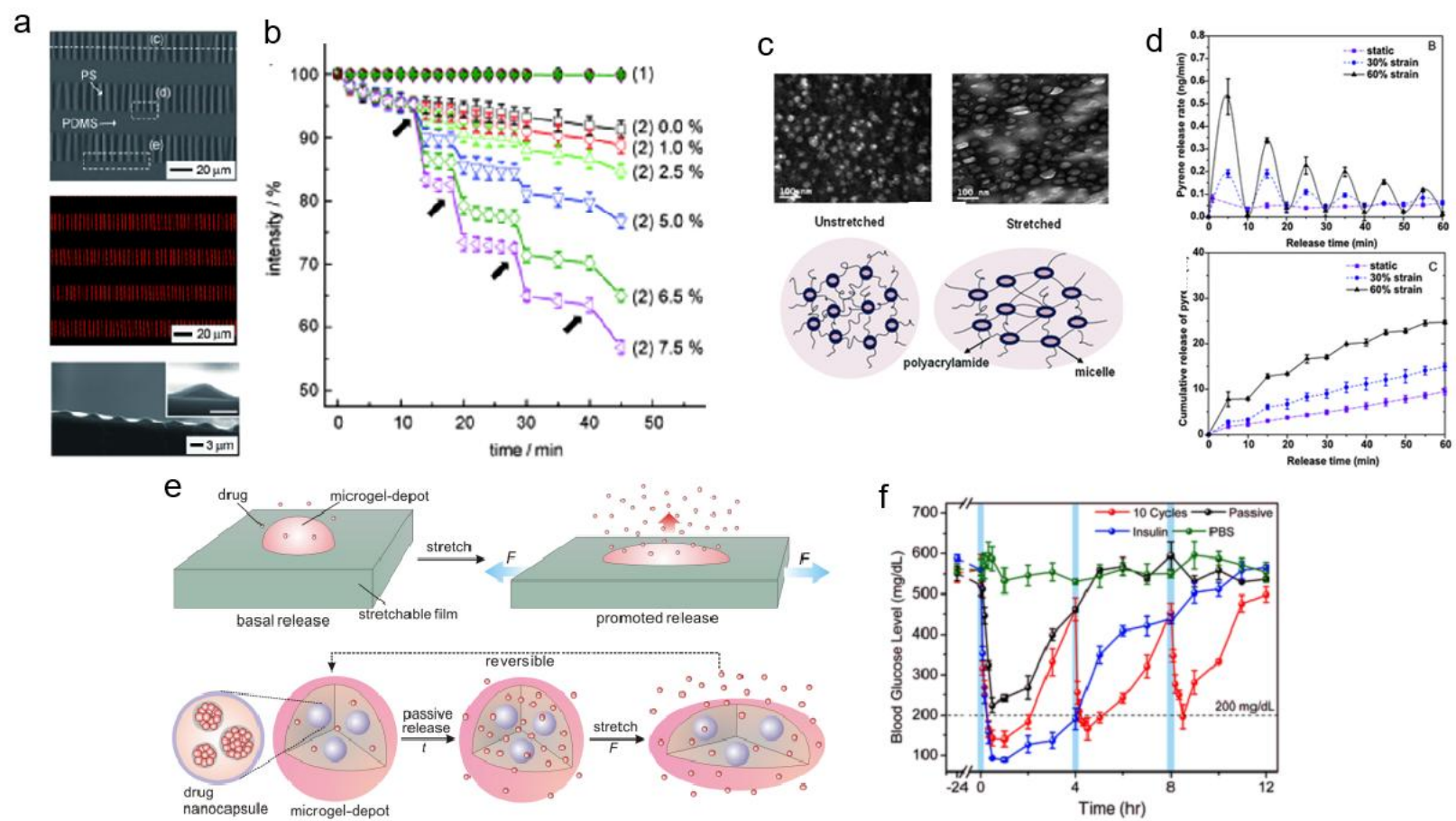

Figure 6. Tensile capsular/ particulate composite systems. (a) Buckled polystyrene films with entrapped rhodamine. (b) Release of rhodamine with increasing tensile strain. (c) Increasing strain deforms pyrene-loaded micelles crosslinked to polyacrylamide matrix to facilitate release. (d) Release rate (top) and cumulative release (bottom) of pyrene with various strains $(0 \%, 30 \%, 60 \%)$. (e) Schematic representation of drugloaded nanoparticles encapsulated in alginate microgel depots, further embedded into an elastomeric film. (f) Decrease in blood glucose levels with the mechanical stimulation of insulin-loaded microneedles in vivo. Figures are reprinted with permission from Refs. [121], [122], and [123].

\subsection{Layered composites}

In contrast to particulate systems, layered composite systems are another strategy to compartmentalize drug encapsulation and to optimize bulk mechanics for tensionresponsive delivery. Arm et al [124] describe the release of bovine albumin or trypsin inhibitor under cyclic tensile stress from poly(lactic-co-glycolic acid) [PLGA] thin film depots wrapped around poly-p-dioxanone cylindrical implants. Using cyclic three-point bending ( $0.4 \mathrm{~Hz}$ (30 mins/ day) for 2 weeks at $0 \mathrm{~mm}, 0.5 \mathrm{~mm}$, or $1 \mathrm{~mm}$ deflections) as a model for loading in long bones, the authors demonstrate faster release rates with larger deflections. Albumin-loaded devices (66-68 $\mathrm{kDa}$ ) release faster than trypsin inhibitor-loaded devices (20 kDa), attributed to enhanced polymer degradation from larger pores left after the larger molecular weight albumin releases. However, this method of loading provides non-uniform stresses and deformations on the implant (higher stresses on outer layers), and thus it is challenging to quantify and compare the necessary stress magnitudes for release. Other approaches for layered composites, including polyelectrolyte films and superhydrophobic coatings, are discussed in the following sections. 


\subsubsection{Polyelectrolyte films}

The use of polyelectrolyte films, consisting of alternating layers of polyanionic and polycationic films, represent another strategy to control permeability by tuning the density of these layers [125-129]. The research groups of Lavalle and Schaaf report tension-responsive delivery systems with polyelectrolyte films that initiate drug/ protein release through: 1) unmasking of active entrapped enzymes; 2) enzymatic modification of entrapped model prodrugs; or 3) enzymatic degradation of the polymeric substrata. The two strata of their systems consist of a thick, low density, exponentially growing polyelectrolyte film (poly (L-lysine)/hyaluronic acid (PLL/HA) that sequesters drug or protein, and a thin, high density, linearly growing (poly(diallyldimethylammonium)/poly(sodium-4-styrenesulfonate) (PDADMA/PSS), or poly(allylamine)/poly(styrene sulfonate) (PAH/PSS)) polyelectrolyte film that acts as a barrier, preventing the permeability of polyelectrolytes and small ions. The two strata are formed on an elastomeric PDMS substrate, capable of withstanding tensile strains up to $100 \%$.

In the first study [130], PDADMA/PSS 5 layers respond to cyclic tensile strain with the opening of nanovalve pores. The visibility of these pores at $50 \%$ strain and $100 \%$ strain led to a follow up study [131] to control the activation of alkaline phosphatase (ALP) from PLL/HA reservoirs, capped by PDADMA/PSS ${ }_{6}$. In the absence of tension, the PDADMA/PSS stratum serves as an effective barrier, impeding the diffusion of ALP by partitioning the enzyme from the substrate, fluorescein diphosphate (FDP). Stretching the system past $70 \%$ critical strain (Figure 8a) thins the PDADMA/PSS layer, uncovering the embedded ALP, allowing dephosphorylation of FDP into fluorescein (F) in the media. Similarly, a subsequent study demonstrates the release and catalysis of fluorescein diphosphate (FDP) from PLL/HA reservoirs, capped by PDADMA/PSS 10 layers containing adsorbed ALP (Figure 8b) [132]. The application of tensile strain past the critical threshold (40\% strain) increases the permeability and diffusion of FDP due to nano-/microscopic structural reorganization of the PDADMA/PSS barrier. The disparity in release from the two systems can be attributed to the difference in molecular size of the two compounds; ALP (enzyme) is much larger than FDP (small molecule) and thus requires stretching to a larger critical strain for initiate release. Consequently, adsorbed ALP catalyzes these freely diffusing FDP molecules into F, leading to an increase in fluorescence intensity based on the strain applied (up to 100\%, Figure 8c). However, the mechanism of release differs in these latter cases unlike the PDADMA/PSS polyelectrolyte layers in the first study, as there are no detectable openings in the PDADMA/PSS ${ }_{6}$ or PDADMA/PSS ${ }_{10}$ layers.

While the above studies demonstrate the stretch-induced release of a model active enzyme and a model prodrug, their follow-up study applies the polyelectrolyte multilayer system to the enzymatic degradation of drug-loaded polymeric films upon application of tension [133]. The mechanosensitive barrier layer consists of a more solid and brittle material, PAH/PSS, which yields openings in the barrier with the application of tension. Trypsin, a protease that recognizes and acts on the C-terminus of lysine residues present in polypeptide chains, enzymatically degrades the PLL/HA reservoir, which is loaded with paclitaxel, a potent mitotic inhibitor chemotherapeutic agent. Without applied tension, the PAH/PSS barrier isolates the reservoir from trypsin and prevents drug release. However, mechanical extension to $30 \%$ strain fractures the barrier, permitting trypsin diffusion into the reservoir through these openings to degrade the reservoir and release fluorescently-labeled paclitaxel. This results in a 7-fold increase in 
fluorescent signal over 8 hours compared to non-stretched devices and devices stretched in the absence of trypsin, confirming the necessity of both stretch and enzymatic activity for drug release (Figure 8d). While the mechanism of release through these openings is explored, the drug release is only evaluated at one tensile strain value $(30 \%)$.

\subsubsection{Wetting of superhydrophobic systems}

Another strategy for constructing mechanoresponsive drug delivery systems relies on the triggered wetting of normally non-wetting or slowly-wetting drug-loaded materials, and is currently used for a variety of biomedical applications [134]. In this manner, water solubilizes the drug, causing subsequent release via diffusion into the surrounding aqueous environment. Mechanically-induced wetting generally employs hydrophobic or superhydrophobic materials with microscopic structural features that become wetted under stimulation [135-137]. Without tension, these features render the material surface non-wetted. Superhydrophobic surfaces, which are characterized by an advancing water contact angle greater than $150^{\circ}$ and low contact angle hysteresis [138,139], are the result of the synergy between a low surface energy material and its rough topology, and applying tension destabilizes this architecture to promote wetting at the material interface.

In contrast to a materials approach to alter wettability through microscale features, the following examples use a more macroscopic approach—specifically, by introducing fractures within composite materials. Huang et al [140] demonstrate the ability to reversibly switch between superhydrophobic and superhydrophilic states with the expansion of various stimuli-responsive acrylamide-based hydrogels coated with silanized glass particles. Increases in tensile strain, $\mathrm{pH}$, or temperature disrupt the superhydrophobic glass particle coating, revealing the underlying hydrophilic hydrogel. Although the authors do not demonstrate release of entrapped agents from the systems, they present control over dye penetration into the alginate-acrylamide hydrogel with increasing strain (up to $600 \%$ ).

Developing a superhydrophobic system for drug delivery, Wang and Kaplan et al control drug release based on tensile strain and further integrate their system with an esophageal stent for ex vivo delivery [141]. In the absence of tensile strain, the superhydrophobic coating, an electrosprayed mixture of biocompatible, biodegradable low surface energy polymers poly( $\varepsilon$-caprolactone) and poly(glycerol monstearate carbonate-co-caprolactone), impedes water infiltration into the hydrophilic drug core (composed of cellulose/polyester), in contrast to a hydrophobic coating of poly $(\varepsilon$ caprolactone) $\left(\right.$ contact angle $=119^{\circ}$ ) (Figure 8e). Increasing strain (at $7 \%$ strain $/ \mathrm{s}$ ) causes greater stress on the superhydrophobic coating, resulting in the development of parallel crack patterns (Figure 8f). The disruption of the coating facilitates the release of entrapped dye (Figure 8e) and chemotherapeutics at strains from $10 \%$ to $100 \%$. The release of chemotherapeutics cisplatin or 7-ethyl-hydroxycamptothecin (which are hydrophilic and hydrophobic, respectively) result in strain-dependent dosing in vitro to OE33 esophageal cancer cells. Finally, expansion of esophageal stents integrated with the tension-responsive coatings enhances the delivery of a fluorescent dye to ex vivo bovine esophagus (Figure $8 \mathrm{~g}$ ). The ability to effectively load and deliver a wide range of compounds (both hydrophilic and hydrophobic) demonstrates the versatility of the system. However, the release under cyclic tension is not evaluated and leaves open the opportunity to design a reversible system in subsequent studies. 
a

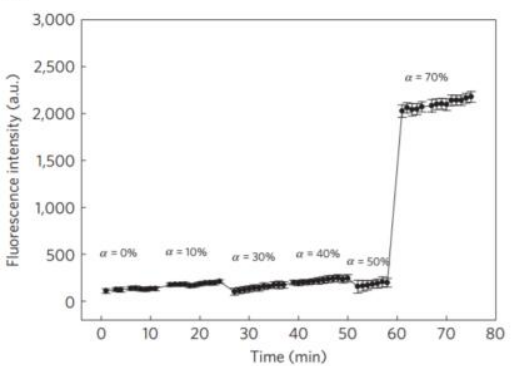

b

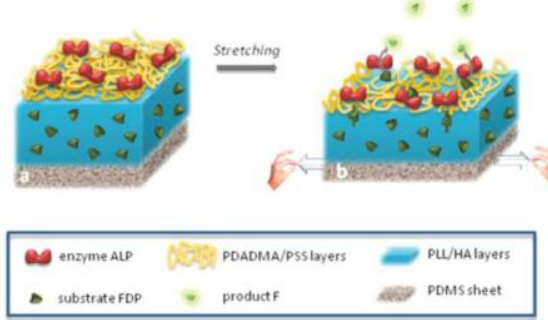

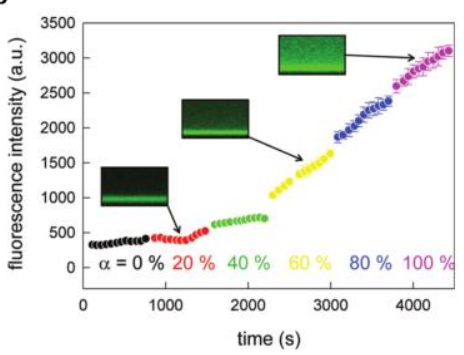

d

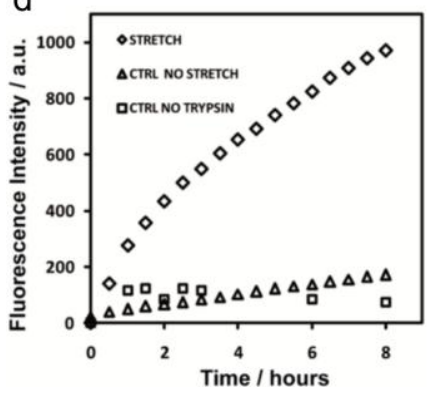

e

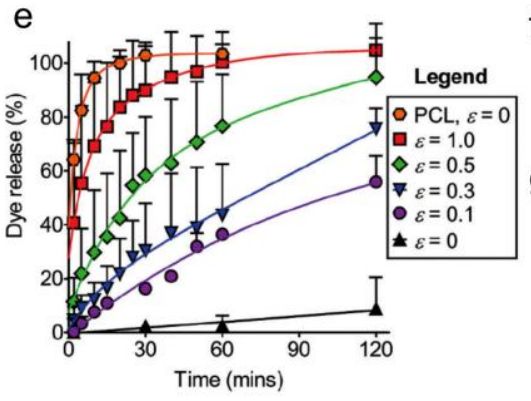

f

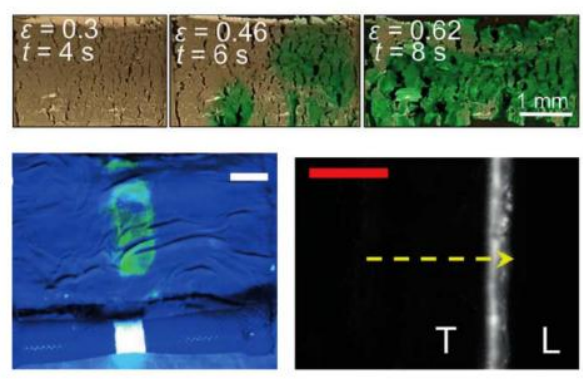

Figure 7. Layered tensile-responsive systems. (a) Increase in enzyme activity results in increased fluorescence past the critical strain (70\%). (b) Schematic representation of FDP release from PLL/HA polyelectrolyte layers and subsequent catalysis by enzyme ALP into fluorescein by stretching. (c) Increase in fluorescence as an indicator of enzymatic activity with increasing tensile strain. (d) Increase in fluorescent intensity with paclitaxel-oregon green release under mechanical stimulation. (e) Modulation of release of dye based on strain from superhydrophobic and hydrophobic composites (PCL). (f) Crack patterns in the superhydrophobic coating with increasing strain facilitate release. (g) System integrated with esophageal stent delivers dye to ex vivo esophageal tissue (under UV light, left) and corresponding cross-section (right, $\mathrm{T}=$ tissue, $\mathrm{L}=$ lumen). Figures are reprinted with permission from Refs. [131], [132], [133], [141]. 


\section{Shear-responsive systems}

Shear-mediated delivery relies on reversible material deformation or disaggregation. Shear forces, present internally or externally (through syringe injections [142]), can trigger release. One major area of focus for shear-responsive delivery is in the cardiovascular system, where narrowing of the vessels increases the local shear stress nearly ten-fold [143,144]. Rather than relying on more conventional biochemical approaches based on targeting moieties (i.e., antibodies), mechanical signals physically target atherosclerotic plaques or clots, as discussed below.

\subsection{Liposome deformation}

In response to high shear environments, liposomes can release their payload due to lipid bilayer flexibility or when in contact with flowing fluids. Natsume and Yoshimoto use this principle to accelerate enzyme activity under shear flow [145]. Glucose oxidase (GO), encapsulated in a three-component liposome system (POPC (1-palmitoyl-2oleoyl-sn-glycero-3-phosphochline), POPG (1-palmitoyl-2-oleoyl-sn-glycero-3phosphoglycerol), and cholesterol), exhibits minimal activity under low shear stress due to the partitioning of the glucose substrate from the enzyme by the liposomal membrane (Figure 8a). With greater laminar shear rates (up to $7.8 \times 10^{3} \mathrm{~s}^{-1}$ ), liposomal membrane permeability increases with $80 \%$ fractional conversion of glucose by GO after 180 minutes compared to $\sim 0 \%$ at zero shear (Figure 8b). The liposome acts as a chaperone for the released GO, preserving the enzymatic activity of free GO even in the presence of empty liposomes, compared to free $\mathrm{GO}$ without liposomes (decreases to $60 \%$ activity after 360 mins) (Figure 8c). Although the system is unique to glucose oxidase, the shear-induced activity of the enzyme is applicable to sensors and as delivery vehicles responsive to shear flow.

Holme et al report another example of using shear to release entrapped agents within 1,3-diamidophospholipid-based vesicles possessing lenticular, rather than spherical, morphologies [146]. The authors hypothesize that spherical vesicles are relatively robust and resistant to mechanical disruption, while the altered morphologies generated by the large bending moduli of modified phospholipid membranes are responsive to elevated shear stress due to breakage along their equators (Figure $8 \mathrm{~d}$ ). Large, unilamellar vesicles, composed of synthetic 1,3-dipalmitamidopropan-2-yl 2(trimethylammonio)ethyl phosphate lipids (Pad-PC-Pad), are prepared with entrapped carboxyfluorescein dye and applied to an in vitro model of atherosclerosis, simulating healthy (i.e., shear stress $=2 \mathrm{~Pa}$ ) and severely constricted arteries (i.e., shear stress $=$ $40 \mathrm{~Pa}$ ). After 40 passes of Pad-PC-Pad through the constricted model, the shearresponsive vesicles release $70 \%$ of entrapped dye compared to only $45 \%$ dye release when passed through the healthy arterial model (Figure 8e). Because the authors focused their efforts on the design and optimization of phospholipid vesicles to achieve shear-responsive release, they left open the opportunity to demonstrate in vivo efficacy in a clinically-relevant model with pharmacologically active agents. 
a

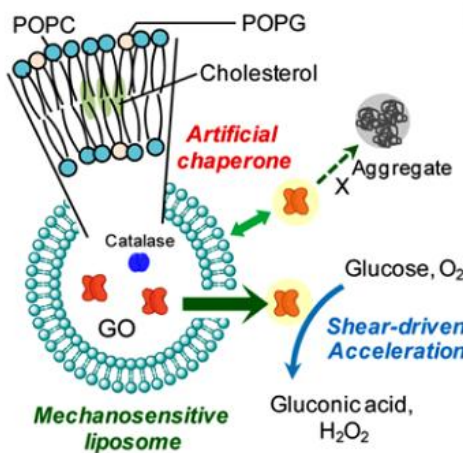

d

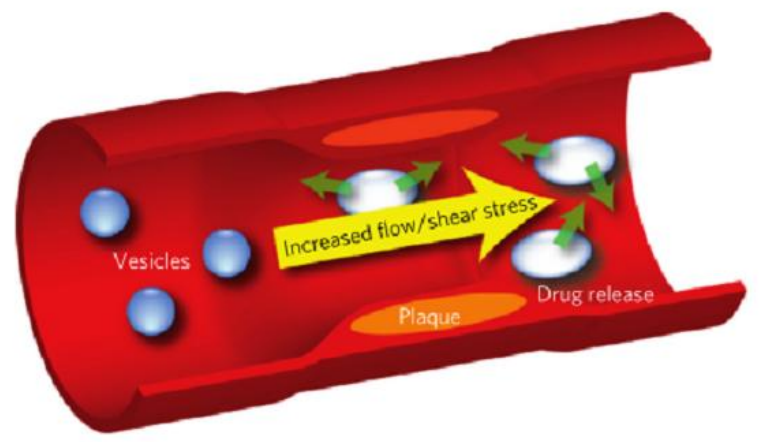

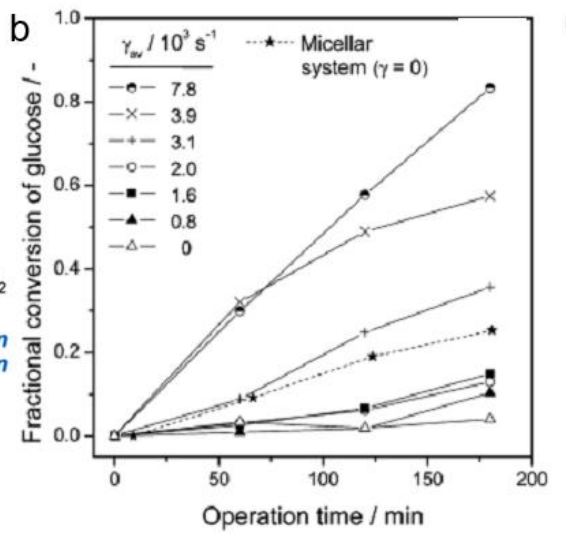

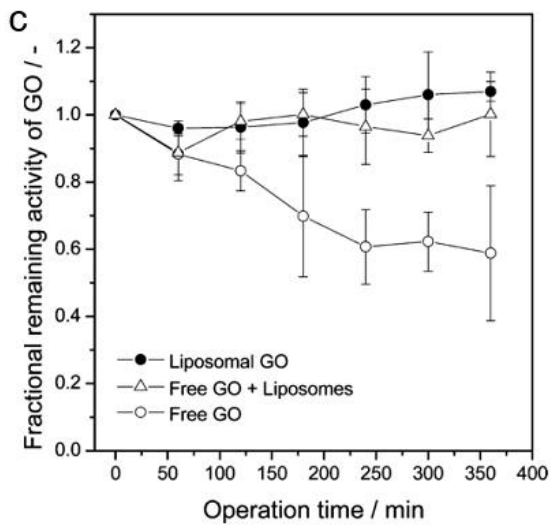

e

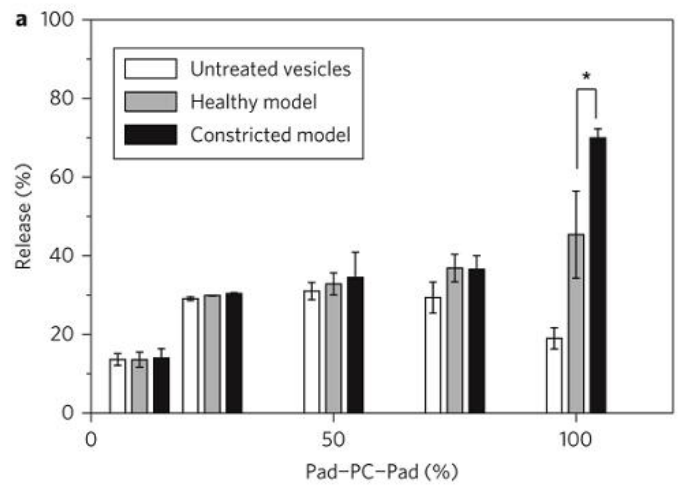

Figure 8. Liposomal shear responsive systems. (a) Schematic representation of liposomal chaperone that increases glucose oxidase activity with increasing shear stress. (b) Corresponding conversion of glucose oxidase with shear. (c) Glucose oxidase activity with encapsulated enzyme, free enzyme, and in the absence of liposomes. (d) Schematic representation of delivery to plaque upon increase in shear stress. (e) Release of dye with untreated vesicles, model healthy arteries, and model constricted arteries. Figures are reprinted with permission from Refs. [145] and [146].

\subsection{Particle aggregation and dispersion}

Microaggregates are capable of dispersing in response to shear stress, offering another strategy for shear-responsive delivery. Korin et al apply their shear-responsive drug delivery system to an elevated shear stress model for cardiovascular disease, and extend their study to an in vivo murine model of myocardial infarction and pulmonary embolism [147]. Nanoparticle microaggregates, composed of poly(lactic-co-glycolic acid) $(50: 50,17 \mathrm{kDa})$, are stable under shear stresses commonly experienced by unobstructed coronary vessels $\left(10-30 \mathrm{dyne} / \mathrm{cm}^{2}\right)$, yet break apart upon experiencing pathologically-relevant shear stresses $\left(>100\right.$ dyne $/ \mathrm{cm}^{2}$ ) for 1 minute, increasing nanoparticle accumulation by nearly 6-fold (Figures 9a, 9b). In a three-dimensional PDMS microfluidic device that simulates a stenosis, the signal from fluorescent nanoparticles increases 16-fold downstream compared to particles with unobstructed flow, and increases 7-fold in cultured endothelial cells downstream compared to cells upstream of the constriction. Finally, nanoparticles formulated with the thrombolytic drug tissue plasminogen activator (tPA) are evaluated for thrombolytic efficacy in a mouse arterial thrombus model. After perfusing preformed fibrin clots, the authors restore pulmonary arterial pressure ex vivo with a 100-fold lower effective dose of tPA (Figure $9 c)$, demonstrating superior performance with the nanoparticle aggregates. Lastly, in an 
in vivo acute thrombus murine model ( 100 clots, $150 \mu \mathrm{m})$, all untreated mice die within 1 hour while $85 \%$ of treated mice survive without symptoms of respiratory distress (Figure 9d). Marosfoi, Korin, and Gounis et al further apply their system in vivo to a large vessel occlusion rabbit model [148]. After complete carotid occlusion, the animals undergo a temporary endovascular bypass, whereby a stent is inserted and expanded at the clot site to temporarily restore high shear blood flow. Compared to stents alone (43\% recanalization) and stents with free recombinant tPA (2 mg and $20 \mathrm{mg}, 14 \%$ and $71 \%$ recanalization, respectively), occlusions treated with stenting and tPA microaggregates are all successfully recanalized.

In contrast, Chen et al use electrostatic forces to adsorb heparin-encapsulated nanoparticles onto red blood cells in order to extend drug circulation time [149]. The nanoparticles, composed of heparin and thiolated poly-L-lysine (PLL) exhibit increased colloidal stability through the formation of disulfide bonds, and are electrostatically attracted to the negatively charged red blood cell surfaces (PLL/ heparin $=6: 5 \mathrm{w} / \mathrm{w},+25$ $\mathrm{mV}$ ). Scanning electron microscope imaging reveals $~ 80 \%$ particle attachment under low shear stress $(1 \mathrm{~Pa})$. However, at high shear-stress $(10 \mathrm{~Pa}), \sim 50 \%$ of the particles remain on the red blood cell after 24 hours, and nearly all particles detach after 48 hours, demonstrating shear-induced nanoparticle detachment.

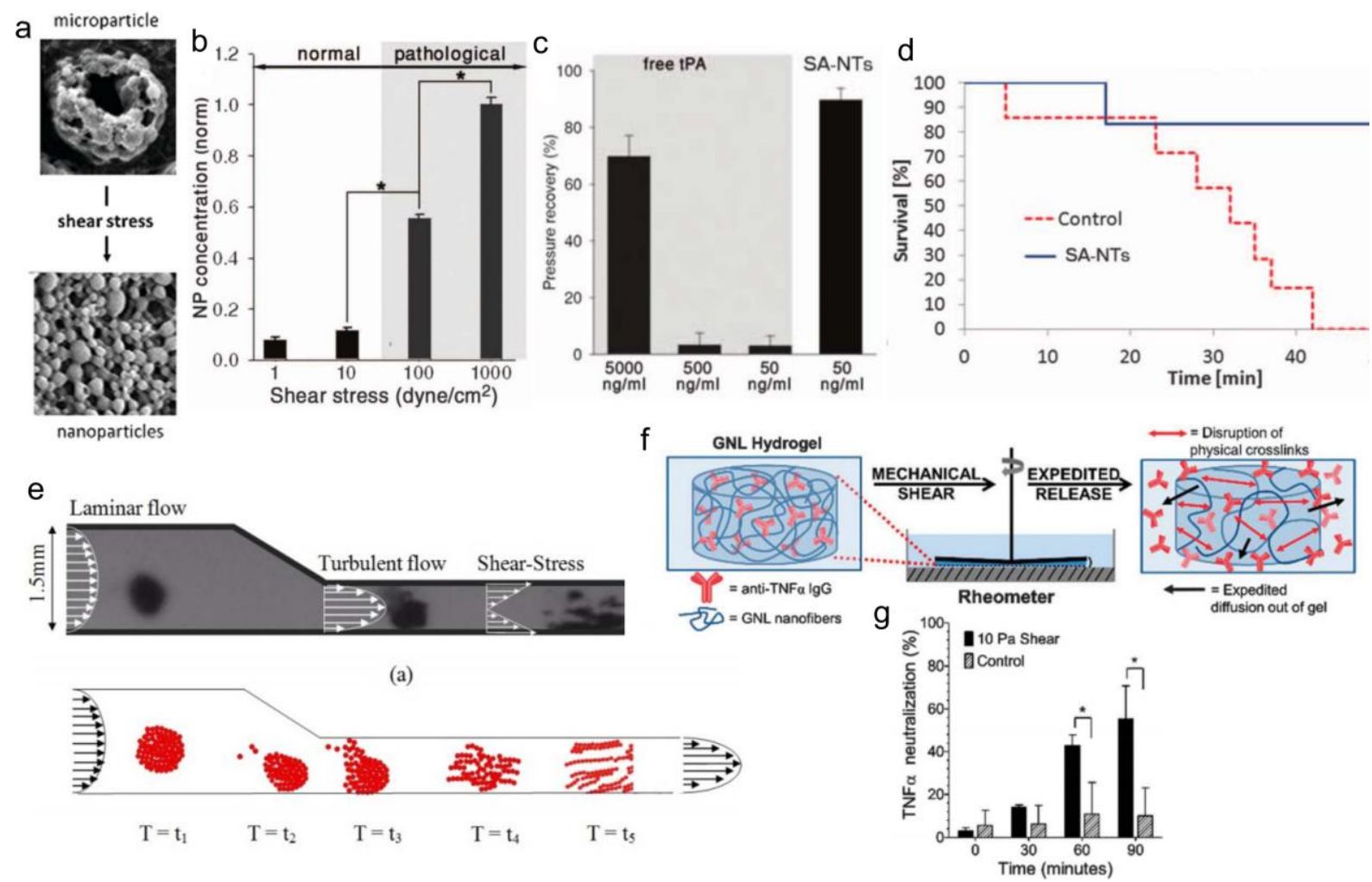

Figure 9. Shear-responsive aggregate systems. (a) Nanoparticle aggregates disperse with increase in shear stress. (b) Increase in nanoparticle accumulation under pathological shear stress compared to normal shear stress. (c) Pressure recovery with 1/100 less dose in nanoparticle aggregates, compared to bolus injection. (d) Survival of mice after treatment in acute thrombus model. (e) Theoretical and experimental dispersion of magnetic iron oxide particles in model artery. (f) Schematic representation of anti-TNFa delivery upon application of shear. (g) Delivery of anti-TNFa and neutralization of TNFa in vitro with increasing shear. Figures are reprinted with permission from Refs. [147], [151], and [152]. 
Anselmo et al mimic another hematocyte, producing platelet-like nanoparticles to target vascular injuries and facilitate hemostasis [150]. These particles, produced by layer-by-layer assemblies of poly(allylamine hydrochloride) (PAH) and bovine serum albumin (BSA), exhibit greater aggregation at higher shear stresses (45 mins at 5, 25, and 55 Dynes $/ \mathrm{cm}^{2}$ ) when surface modified with collagen-binding peptides (CBP) and von Willebrand Factor (VWF) binding peptides (VBP) (for wound targeting) on a collagen/ vWF coated glass slide, or surface modified with fibrinogen-mimetic peptide (FMP, for platelet binding) on glass slides with activated platelets, compared to particles lacking surface modifications or surface modified particles on BSA-coated glass slides. The authors also demonstrate greater particle aggregations after flowing the particles through a glass capillary. This shear strain-dependent aggregation, similar to physiological platelets encountering a site of vascular injury, is attributed to the interaction of FMP and the flexible discoid shape that concentrates particles to vessel walls. In a tail transection mouse model, the CBP, VBP, and FMP functionalized particles decrease bleeding time by $\sim 65 \%$. Analysis of the subsequent tail clots verify the incorporation of nanoparticle aggregates, as predicted in vitro, and their ability to expedite the formation of hemostatic plugs.

In another approach, Mellal et al report the dispersion of therapeutic MRI (magnetic resonance imaging)-guided magnetic microcarriers to deliver site-specific drug [151]. Simulating flow through an artery, computational models demonstrate parameters to maximize drug loading with superparamagnetic iron oxide particle aggregates while maintaining the ability to navigate in arterial blood flow. Specifically, chain-like aggregations exhibit higher magnetic volume, to steer more efficiently, than spherical or ellipsoid-like aggregates. The results are experimentally verified with flow through a microfluidic arterial bifurcation using 3D Maxwell-Helmholtz coils. Figure 9e shows the evolution of the aggregates under high shear stress (simulating $60 \%$ vessel occlusion) from spherical to ellipsoidal-like to chain-like structures, in accordance to experimental and theoretical data. With increasing shear force, the aggregation volume to the particle volume decreases by nearly half, disrupting the magnetic dipole-dipole forces. The disruption of the aggregate downstream releases drug and allows facile clearance of particles from the body. While the computational data supports the utility of aggregates, the studies do not show drug distribution after release nor does the experimental data demonstrate efficacious drug delivery.

\subsection{Supramolecular disassembly}

Self-assembled structures exhibit reversible or self-healing characteristics for shearresponsive delivery. Kaplan et al report the delivery of anti-TNFa antibody upon the application of shear stress from a self-assembled hydrogel [152]. The supramolecular nanofiber gel, composed of sugar, nucleoside, and fatty acid subunits, disassembles under increasing shear stress (up to $\sim 27 \mathrm{~Pa}$ ) and reforms within 2 minutes of the removal of shear. The nanofiber assembly slows the diffusion of entrapped large macromolecules in a weight-dependent manner (e.g., 19.6 kD FITC-dextran diffuses out more than twice as fast as $167 \mathrm{kD}$ FITC-dextran). The shear-induced disassembly of the gel expedites the release of anti-TNFa antibody (Figure 9f). The resulting release from 90 minutes of $10 \mathrm{~Pa}$ shear stress reduces TNFa toxicity in vitro by $60 \%$ in L292 cells, a murine TNFa-sensitive fibroblast cell line (Figure 9g). The delivery of anti-TNFa antibody would serve as an anti-inflammatory treatment for diseases, such as rheumatoid arthritis, effectively neutralizing the effects of TNFa. 


\section{Conclusions and future perspectives}

The field of mechanically-stimulated delivery is rapidly expanding with a number of reports demonstrating the promise of releasing pharmaceutical/ active agents in a controlled manner. These mechanoresponsive systems are designed to be clinically relevant through physiological force triggers or externally applied clinical devices. The dynamic nature of the human body is constantly subjected to forces; therefore determining triggers distinguishable from the mechanical forces routinely present in daily activities are key to targeting and maximizing release.

To take advantage of compressive forces, investigators are exploring drug loaded porous polymer scaffolds, siloxanes, hydrogels, crosslinked micellular gels, and layerby-layer microparticles. Key features reported in these systems are: 1) mechanical integrity to withstand and respond to multiple compressions, 2) incorporation of molecular containers or micelles for greater loading of hydrophobic agents, and 3) dose dependent cyclic release. Since the first report in the late 1980s, two of nine delivery systems have evaluated performance in vivo: a two-component silicone implant that responds to compression to successfully deliver insulin, thereby reducing glucose levels in diabetic rats [99]; and a VEGF-loaded calcium-crosslinked hydrogel that increases vascularization upon cyclic compression [103].

Tension-responsive systems utilize stretchable hydrogels, siloxane substrates, crosslinkable micelles, polyelectrolyte layer-by-layer films, and superhydrophobic composites. These systems possess: 1) the mechanical strength to be subjected to high tensile strains, 2) capsular or layered composites to encapsulate drugs and proteins, and 3) cycle- or strain-dependent release. Since the first report in 1997, only one delivery system out of nine has evaluated performance in vivo (stretched microneedles containing insulin microparticles prolong delivery in diabetic mice [123]) and only one other delivery system has evaluated performance ex vivo (drug release from a superhydrophobic composite is triggered by the expansion of the device, integrated with an esophageal stent, in ex vivo bovine esophagus [141]).

In contrast, shear-activated systems exhibit: 1) reversible material deformation via liposomes, 2) disaggregation of microparticles or nanofibers to release their payload, or 3 ) accumulation of aggregates at the target site. Well-characterized increases in physiological forces, such as the increase in shear stress noted at strictures in the cardiovascular system [143,144], act as both viable mechanical triggers and drug targets. At the time of this publication, there are three reports of in vivo assessment: the delivery of tissue plasminogen activator that dissolves clots in both an acute thrombus murine model and a large vessel occlusion rabbit model $[147,148]$, and the aggregation of platelet mimics that decrease in bleeding time in a tail transection mouse model [150].

Overall, in comparison to other stimuli-responsive systems, there are only a handful of reports on the mechanoresponsive delivery of active therapeutics. The ease of characterization with model dyes or compounds demonstrates proof-of-concept, but to translate mechanoresponsive systems to the clinic, the delivery of therapeutic agents in vivo will need to be further developed. Currently, there are no mechanically-activated drug delivery devices approved for use in patients, although numerous opportunities

exist for new designs of drug-device combinations to enhance treatment of cardiovascular, musculoskeletal, and pulmonary diseases. For example, the increase in stress within vessels containing atherosclerotic plaques and arterial embolisms allow 
physical rather than biochemical targeting to stenotic sites for drug release. Liposomes and microaggregates deform and disaggregate, respectively, under a higher shear environment to release their payloads. Treatment can also be enhanced by integrating mechanoresponsive drug or protein delivery systems with existing mechanical medical devices, such as stents and catheters. The radial expansion of esophageal stents, which treat esophageal cancer, acts as a trigger to control delivery of chemotherapeutics [141]. Other systems offer new mechanical approaches to treatment; user-controlled delivery of drugs and proteins are reported for pain management, neoangiogenesis, and diabetes.

While we report these promising mechanoresponsive examples, many opportunities and challenges exist for the future of these systems. Current compressive systems, composed of elastomeric substrates and hydrogels, successfully withstand repeated compressions and will need to be continually developed to maximize agent encapsulation and minimize release in the absence of compression. The ease of externally applying these compressive forces on implanted systems has resulted in increased interest in these systems, but improved characterization and precise application of these forces are needed to reduce the variability in dosing.

Similarly, well-characterized tensile forces should be used as triggers. The robust characterization and increasing deployment of mechanical medical devices, such as balloon catheters and stents, has led to their use as viable triggers to further enhance current medical care. Towards these applications, tension-responsive systems should tolerate higher strains $(>30 \%)$, and this has been demonstrated in many of the current systems using hydrogels, siloxanes, and polyester meshes. The problems encountered with first generation systems, such as limited loading of agents and diffusive release in the absence of tensile triggers, are being resolved through capsular and layered composites.

In contrast, shear-responsive systems rely on the reversible deformation of lipids in liposomes, or the dispersion of microparticle or nanofiber aggregates to release the loaded therapeutics. The majority of reports on shear-responsive delivery focus on cardiovascular indications (i.e., atherosclerosis) as the increase in shear stress, due to narrowing of blood vessels, provides a pathophysiological trigger for site-specific delivery.

The diversity of active therapeutic agents, as opposed to model dyes and compounds, investigated in shear, tension, and compression activated system is minimal in the compositional and structural space. For example, there are no examples of nucleic acids being delivered by such systems, and delivery of only one type of growth factor (VEGF [103]), one type of antibody (anti-TNF $\alpha$ [152]), three proteins/ enzymes (insulin [99,123], glucose oxidase [145], tissue plasminogen activator $[147,148]$ ), one polysaccharide (heparin [149]), and seven small molecules (ondansetron [106], hydrocortisone [107], dexamethasone [108], doxorubicin [123], paclitaxel [133], cisplatin [141], and camptothecin [141]) have been described. Delivery is further complicated by the necessity to preserve the activity of small molecules and proteins within the mechanoreponsive systems. Lastly, in all these cases long-term assessments of implant materials and the development of biodegradable substrates are needed before clinical translation becomes feasible.

The various systems described utilize a number of different delivery strategies, from inducing flux through increased pressure (hydrogels, elastomers under compression) to 
increasing the available surface area or porosity to expedite diffusion (liposomes, hydrogels, elastomers under shear/ tension). Many of the release compounds are not chemically conjugated to the matrix; instead they are physically incorporated and thus rely on a diffusive gradient increase to exudate compounds. One exception to this case is the mechanochemical activation of oxanorbornadiene bonds, where the application of physical force breaks chemical bonds to release, albeit also limits release to, a furanderivative [102]. However, the reliance on diffusion for many of these systems has led to strategies to prolong drug release. Inclusion complexes [106,107], micelles [108,153], and particles $[100,123,147,149]$ increase loading of hydrophobic compounds while also allowing retention for a longer time period in the absence of mechanical stimuli. Furthermore, as drug carrier systems, they act as an additional rate-limiting step to compound release. In contrast, other studies have sought to expedite release. In one example, stretching exposes the drug reservoir to enzymatic degradation of the matrix, resulting in release. Nevertheless, much of the release kinetics also depends on the physical properties of the drug and their dissolution into release media. Of the 26 studies, only three have reported using compounds of varying hydrophobicities, charge, or molecular weight $[109,121,141]$. In general, there is higher loading and release of small neutral molecules, compared to charged or higher molecular weight compounds. Studying various release compounds is especially critical for systems that utilize mechanical targets (i.e., increasing shear at vessel strictures) to determine the optimal drug with the proper dosing.

The examples highlighted in this review demonstrate both the capabilities and limitations of mechanically-activated systems. Challenges for many of the mechanoresponsive delivery systems reported thus far are a) response over a limited range and b) struggling to prevent release (i.e., burst release or leaky release) in the absence of stimuli. Additionally, many of these systems utilize large forces to trigger drug release and thus materials that can respond to smaller forces (e.g., cellular) represent an unexplored and critical area of research. Harnessing mechanical forces, either internal or external, to control the release of active agents in vivo is a viable strategy for drug delivery with significant clinical promise in the near future.

The motivation for writing this review manuscript is to recognize the research achievements to date, to stimulate discovery of new mechanoresponsive delivery designs and device compositions, and to encourage all to work in this exciting area of drug delivery.

\section{Acknowledgements}

The authors thank the National Institutes of Health (T32 EB006359, M.W.G., J.W.), the National Science Foundation (DGE-1247312, J.W.; DMR-1507081, M.W.G), and the Wallace H. Coulter Translational Research Partnership Award (M.W.G., Y.L.C., J.W.) for support.

\section{Author Contributions}

J.W., J.A.K., Y.L.C., and M.W.G. wrote the manuscript. All authors reviewed the manuscript. All authors have given approval to the final version of the manuscript. 


\section{References}

[1] D. Schmaljohann, Thermo- and pH-responsive polymers in drug delivery., Adv. Drug Deliv. Rev. 58 (2006) 1655-70. doi:10.1016/j.addr.2006.09.020.

[2] J. Liu, Y. Huang, A. Kumar, A. Tan, S. Jin, A. Mozhi, et al., pH-sensitive nanosystems for drug delivery in cancer therapy., Biotechnol. Adv. 32 (2014) 693-710. doi:10.1016/j.biotechadv.2013.11.009.

[3] X. He, J. Li, S. An, C. Jiang, pH-sensitive drug-delivery systems for tumor targeting., Ther. Deliv. 4 (2013) 1499-510. doi:10.4155/tde.13.120.

[4] W. Gao, J.M. Chan, O.C. Farokhzad, pH-Responsive nanoparticles for drug delivery., Mol. Pharm. 7 (2010) 1913-20. doi:10.1021/mp100253e.

[5] Y.-J. Zhu, F. Chen, pH-responsive drug-delivery systems., Chem. Asian J. 10 (2015) 284-305. doi:10.1002/asia.201402715.

[6] P. Gupta, K. Vermani, S. Garg, Hydrogels: from controlled release to $\mathrm{pH}$ responsive drug delivery, Drug Discov. Today. 7 (2002) 569-579. doi:10.1016/S1359-6446(02)02255-9.

[7] Y.L. Colson, M.W. Grinstaff, Biologically Responsive Polymeric Nanoparticles for Drug Delivery, Adv. Mater. 24 (2012) 3878-3886. doi:10.1002/adma.201200420.

[8] A.P. Griset, J. Walpole, R. Liu, A. Gaffey, Y.L. Colson, M.W. Grinstaff, Expansile Nanoparticles: Synthesis, Characterization, and in Vivo Efficacy of an AcidResponsive Polymeric Drug Delivery System, J. Am. Chem. Soc. 131 (2009) 2469-2471. doi:10.1021/ja807416t.

[9] H. Yuan, B. Li, K. Liang, X. Lou, Y. Zhang, Regulating drug release from $\mathrm{pH}$ - and temperature-responsive electrospun CTS-g-PNIPAAm/poly(ethylene oxide) hydrogel nanofibers., Biomed. Mater. 9 (2014) 55001. doi:10.1088/17486041/9/5/055001.

[10] M. Constantin, S. Bucatariu, V. Harabagiu, I. Popescu, P. Ascenzi, G. Fundueanu, Poly(N-isopropylacrylamide-co-methacrylic acid) $\mathrm{pH} /$ thermoresponsive porous hydrogels as self-regulated drug delivery system., Eur. J. Pharm. Sci. 62 (2014) 86-95. doi:10.1016/j.ejps.2014.05.005.

[11] Y. Koyamatsu, T. Hirano, Y. Kakizawa, F. Okano, T. Takarada, M. Maeda, pHresponsive release of proteins from biocompatible and biodegradable reverse polymer micelles., J. Control. Release. 173 (2014) 89-95. doi:10.1016/j.jconrel.2013.10.035.

[12] S.D. Fitzpatrick, L.E. Fitzpatrick, A. Thakur, M.A.J. Mazumder, H. Sheardown, Temperature-sensitive polymers for drug delivery., Expert Rev. Med. Devices. 9 (2012) 339-51. doi:10.1586/erd.12.24.

[13] X. Gu, J. Wang, X. Liu, D. Zhao, Y. Wang, H. Gao, et al., Temperature-responsive drug delivery systems based on polyaspartamides with isopropylamine pendant groups, Soft Matter. 9 (2013) 7267. doi:10.1039/c3sm50904d.

[14] J.. Chung, M. Yokoyama, M. Yamato, T. Aoyagi, Y. Sakurai, T. Okano, Thermoresponsive drug delivery from polymeric micelles constructed using block copolymers of poly( $\mathrm{N}$-isopropylacrylamide) and poly(butylmethacrylate), J. Control. Release. 62 (1999) 115-127. doi:10.1016/S0168-3659(99)00029-2.

[15] L. Bromberg, Temperature-responsive gels and thermogelling polymer matrices for protein and peptide delivery, Adv. Drug Deliv. Rev. 31 (1998) 197-221. doi:10.1016/S0169-409X(97)00121-X.

[16] D. Needham, M.W. Dewhirst, The development and testing of a new temperature- 
sensitive drug delivery system for the treatment of solid tumors, Adv. Drug Deliv. Rev. 53 (2001) 285-305. doi:10.1016/S0169-409X(01)00233-2.

[17] N. Hijnen, S. Langereis, H. Grüll, Magnetic resonance guided high-intensity focused ultrasound for image-guided temperature-induced drug delivery., Adv. Drug Deliv. Rev. 72 (2014) 65-81. doi:10.1016/j.addr.2014.01.006.

[18] K. Ninomiya, S. Kawabata, H. Tashita, N. Shimizu, Ultrasound-mediated drug delivery using liposomes modified with a thermosensitive polymer., Ultrason. Sonochem. 21 (2014) 310-6. doi:10.1016/j.ultsonch.2013.07.014.

[19] Q. He, J. Liu, C. Huang, Z. Wu, A Nanoscale System for Remarkably Enhanced Drug Delivery Based on Hollow Magnetic Particles Encapsulated Within Temperature-Responsive Poly(methylmethacrylate), Sci. Adv. Mater. 6 (2014) 387-398. doi:10.1166/sam.2014.1728.

[20] B.P. Timko, M. Arruebo, S.A. Shankarappa, J.B. McAlvin, O.S. Okonkwo, B. Mizrahi, et al., Near-infrared-actuated devices for remotely controlled drug delivery., Proc. Natl. Acad. Sci. U. S. A. 111 (2014) 1349-54. doi:10.1073/pnas.1322651111.

[21] Z. Yu, N. Li, P. Zheng, W. Pan, B. Tang, Temperature-responsive DNA-gated nanocarriers for intracellular controlled release., Chem. Commun. (Camb). 50 (2014) 3494-7. doi:10.1039/c3cc49183h.

[22] J.G. Christie, U.B. Kompella, Ophthalmic light sensitive nanocarrier systems., Drug Discov. Today. 13 (2008) 124-34. doi:10.1016/j.drudis.2007.12.005.

[23] P. Shum, J.-M. Kim, D.H. Thompson, Phototriggering of liposomal drug delivery systems, Adv. Drug Deliv. Rev. 53 (2001) 273-284. doi:10.1016/S0169409X(01)00232-0.

[24] W. Cheng, L. Gu, W. Ren, Y. Liu, Stimuli-responsive polymers for anti-cancer drug delivery., Mater. Sci. Eng. C. Mater. Biol. Appl. 45 (2014) 600-8. doi:10.1016/j.msec.2014.05.050.

[25] J. Lu, E. Choi, F. Tamanoi, J.I. Zink, Light-activated nanoimpeller-controlled drug release in cancer cells., Small. 4 (2008) 421-6. doi:10.1002/smll.200700903.

[26] C. Alvarez-Lorenzo, L. Bromberg, A. Concheiro, Light-sensitive Intelligent Drug Delivery Systems, Photochem. Photobiol. 85 (2009) 848-860. doi:10.1111/j.17511097.2008.00530.x.

[27] P. Markland, Y. Zhang, G.L. Amidon, V.C. Yang, A pH- and ionic strengthresponsive polypeptide hydrogel: synthesis, characterization, and preliminary protein release studies., J. Biomed. Mater. Res. 47 (1999) 595-602. doi:10.1002/(SICl)1097-4636(19991215)47:4<595::AID-JBM17>3.0.CO;2-I.

[28] R. Zhang, M. Tang, A. Bowyer, R. Eisenthal, J. Hubble, A novel pH- and ionicstrength-sensitive carboxy methyl dextran hydrogel., Biomaterials. 26 (2005) 4677-83. doi:10.1016/j.biomaterials.2004.11.048.

[29] K. Järvinen, S. Åkerman, B. Svarfvar, T. Tarvainen, P. Viinikka, P. Paronen, Drug Release from $\mathrm{pH}$ and lonic Strength Responsive Poly(acrylic acid) Grafted Poly(vinylidenefluoride) Membrane Bags In Vitro, Pharm. Res. 15 (n.d.) 802-805. doi:10.1023/A:1011995725320.

[30] R. V Kulkarni, S. Biswanath, Electrically responsive smart hydrogels in drug delivery: a review., J. Appl. Biomater. Biomech. 5 125-39. http://europepmc.org/abstract/med/20799182 (accessed June 2, 2016).

[31] S. Murdan, Electro-responsive drug delivery from hydrogels, J. Control. Release. 92 (2003) 1-17. doi:10.1016/S0168-3659(03)00303-1. 
[32] J. Ge, E. Neofytou, T.J. Cahill, R.E. Beygui, R.N. Zare, Drug release from electricfield-responsive nanoparticles., ACS Nano. 6 (2012) 227-33. doi:10.1021/nn203430m.

[33] V. Pillay, T.-S. Tsai, Y.E. Choonara, L.C. du Toit, P. Kumar, G. Modi, et al., A review of integrating electroactive polymers as responsive systems for specialized drug delivery applications., J. Biomed. Mater. Res. A. 102 (2014) 2039-54. doi:10.1002/jbm.a.34869.

[34] S.Y. Kim, Y.M. Lee, Drug release behavior of electrical responsive poly(vinyl alcohol)/poly(acrylic acid) IPN hydrogels under an electric stimulus, J. Appl. Polym. Sci. 74 (1999) 1752-1761. doi:10.1002/(SICI)10974628(19991114)74:7<1752::AID-APP18>3.0.CO;2-H.

[35] I.C. Kwon, Y.H. Bae, S.W. Kim, Electrically erodible polymer gel for controlled release of drugs., Nature. 354 (1991) 291-3. doi:10.1038/354291a0.

[36] A. Servant, C. Bussy, K. Al-Jamal, K. Kostarelos, Design, engineering and structural integrity of electro-responsive carbon nanotube- based hydrogels for pulsatile drug release, J. Mater. Chem. B. 1 (2013) 4593. doi:10.1039/c3tb20614a.

[37] P.M. George, D.A. LaVan, J.A. Burdick, C.-Y. Chen, E. Liang, R. Langer, Electrically Controlled Drug Delivery from Biotin-Doped Conductive Polypyrrole, Adv. Mater. 18 (2006) 577-581. doi:10.1002/adma.200501242.

[38] U.O. Häfeli, Magnetically modulated therapeutic systems., Int. J. Pharm. 277 (2004) 19-24. doi:10.1016/j.ijpharm.2003.03.002.

[39] J. Cheng, B.A. Teply, S.Y. Jeong, C.H. Yim, D. Ho, I. Sherifi, et al., Magnetically responsive polymeric microparticles for oral delivery of protein drugs., Pharm. Res. 23 (2006) 557-64. doi:10.1007/s11095-005-9444-5.

[40] T. Hoare, J. Santamaria, G.F. Goya, S. Irusta, D. Lin, S. Lau, et al., A magnetically triggered composite membrane for on-demand drug delivery., Nano Lett. 9 (2009) 3651-7. doi:10.1021/n19018935.

[41] T. Kubo, T. Sugita, S. Shimose, Y. Nitta, Y. Ikuta, T. Murakami, Targeted delivery of anticancer drugs with intravenously administered magnetic liposomes in osteosarcoma-bearing hamsters., Int. J. Oncol. 17 (2000) 309-324. http://www.spandidos-publications.com/ijo/17/2/309/abstract (accessed June 2, 2016).

[42] C.S.S.R. Kumar, F. Mohammad, Magnetic nanomaterials for hyperthermia-based therapy and controlled drug delivery., Adv. Drug Deliv. Rev. 63 (2011) 789-808. doi:10.1016/j.addr.2011.03.008.

[43] E. Muntimadugu, A. Jain, W. Khan, Targeted Drug Delivery Systems: Strategies and Challenges, Springer International Publishing, 2015. doi:10.1007/978-3-31911355-5.

[44] I.J. Bruvera, R. Hernández, C. Mijangos, G.F. Goya, An integrated device for magnetically-driven drug release and in situ quantitative measurements: Design, fabrication and testing, J. Magn. Magn. Mater. 377 (2015) 446-451. doi:10.1016/j.jmmm.2014.10.149.

[45] L.-A. Tziveleka, P. Bilalis, A. Chatzipavlidis, N. Boukos, G. Kordas, Development of multiple stimuli responsive magnetic polymer nanocontainers as efficient drug delivery systems., Macromol. Biosci. 14 (2014) 131-41. doi:10.1002/mabi.201300212.

[46] L. Zha, B. Banik, F. Alexis, Stimulus responsive nanogels for drug delivery, Soft 
Matter. 7 (2011) 5908. doi:10.1039/c0sm01307b.

[47] S. Mura, J. Nicolas, P. Couvreur, Stimuli-responsive nanocarriers for drug delivery., Nat. Mater. 12 (2013) 991-1003. doi:10.1038/nmat3776.

[48] L. Dong, H. Peng, S. Wang, Z. Zhang, J. Li, F. Ai, et al., Thermally and magnetically dual-responsive mesoporous silica nanospheres: preparation, characterization, and properties for the controlled release of sophoridine, J. Appl. Polym. Sci. 131 (2014) n/a-n/a. doi:10.1002/app.40477.

[49] C.T. Lim, E.H. Zhou, S.T. Quek, Mechanical models for living cells--a review., J. Biomech. 39 (2006) 195-216. doi:10.1016/j.jbiomech.2004.12.008.

[50] D.E. Ingber, Tensegrity II. How structural networks influence cellular information processing networks, J. Cell Sci. 116 (2003) 1397-1408. doi:10.1242/jcs.00360.

[51] M.A. Schwartz, A.W. Orr, B.P. Helmke, B.R. Blackman, M.A. Schwartz, A.D. Bershadsky, et al., Cell biology. The force is with us., Science. 323 (2009) 588-9. doi:10.1126/science.1169414.

[52] S.E. Mutsaers, J.E. Bishop, G. McGrouther, G.J. Laurent, Mechanisms of tissue repair: from wound healing to fibrosis, Int. J. Biochem. Cell Biol. 29 (1997) 5-17. doi:10.1016/S1357-2725(96)00115-X.

[53] R. Agha, R. Ogawa, G. Pietramaggiori, D.P. Orgill, A Review of the Role of Mechanical Forces in Cutaneous Wound Healing, J. Surg. Res. 171 (2011) 700 708. doi:10.1016/j.jss.2011.07.007.

[54] D. Ingber, Mechanobiology and diseases of mechanotransduction, Ann. Med. 35 (2003) 564-577. doi:10.1080/07853890310016333.

[55] N.S. Sverdlova, U. Witzel, Principles of determination and verification of muscle forces in the human musculoskeletal system: Muscle forces to minimise bending stress., J. Biomech. 43 (2010) 387-96. doi:10.1016/j.jbiomech.2009.09.049.

[56] A. Erdemir, S. McLean, W. Herzog, A.J. van den Bogert, Model-based estimation of muscle forces exerted during movements., Clin. Biomech. (Bristol, Avon). 22 (2007) 131-54. doi:10.1016/j.clinbiomech.2006.09.005.

[57] S. Glagov, C. Zarins, D.P. Giddens, D.N. Ku, Hemodynamics and atherosclerosis. Insights and perspectives gained from studies of human arteries., Arch. Pathol. Lab. Med. 112 (1988) 1018-31. http://europepmc.org/abstract/med/3052352 (accessed April 11, 2016).

[58] M. Zamir, Shear forces and blood vessel radii in the cardiovascular system, J. Gen. Physiol. 69 (1977) 449-461. doi:10.1085/jgp.69.4.449.

[59] R.S. Reneman, T. Arts, A.P.G. Hoeks, Wall Shear Stress - an Important Determinant of Endothelial Cell Function and Structure - in the Arterial System in vivo, J. Vasc. Res. 43 (2006) 251-269. doi:10.1159/000091648.

[60] J. MEAD, Mechanical properties of lungs., Physiol. Rev. 41 (1961) 281-330. http://www.ncbi.nlm.nih.gov/pubmed/13768766 (accessed June 9, 2016).

[61] A. RUBINI, V. CATENA, D. DEL MONTE, E.L. CARNIEL, A REVIEW OF THE EFFECTS OF BODY TEMPERATURE VARIATIONS ON RESPIRATORY MECHANICS: MEASUREMENTS BY THE END-INFLATION OCCLUSION METHOD IN THE RAT, J. Mech. Med. Biol. 15 (2015) 1530006. doi:10.1142/S0219519415300069.

[62] W. Wu, W.-Q. Wang, D.-Z. Yang, M. Qi, Stent expansion in curved vessel and their interactions: a finite element analysis., J. Biomech. 40 (2007) 2580-5. doi:10.1016/j.jbiomech.2006.11.009.

[63] J. de Ribamar Costa, G.S. Mintz, S.G. Carlier, K. Fujii, K. Sano, M. Kimura, et al., 
Intravascular ultrasound assessment of drug-eluting stent expansion., Am. Heart J. 153 (2007) 297-303. doi:10.1016/j.ahj.2006.08.026.

[64] C. Lally, F. Dolan, P.J. Prendergast, Cardiovascular stent design and vessel stresses : a finite element analysis, 38 (2005) 1574-1581.

doi:10.1016/j.jbiomech.2004.07.022.

[65] F. Migliavacca, L. Petrini, M. Colombo, F. Auricchio, R. Pietrabissa, Mechanical behavior of coronary stents investigated through the finite element method, J. Biomech. 35 (2002) 803-811. doi:10.1016/S0021-9290(02)00033-7.

[66] J.A. Goldstein, J.S. Barkin, Comparison of the diameter consistency and dilating force of the controlled radial expansion balloon catheter to the conventional balloon dilators., Am. J. Gastroenterol. 95 (2000) 3423-7. doi:10.1111/j.15720241.2000.03357.x.

[67] J.B. Simpson, D.S. Baim, E.W. Robert, D.C. Harrison, A new catheter system for coronary angioplasty, Am. J. Cardiol. 49 (1982) 1216-1222. doi:10.1016/00029149(82)90047-9.

[68] Y. Zhang, J. Yu, H.N. Bomba, Y. Zhu, Z. Gu, Mechanical Force-Triggered Drug Delivery, Chem. Rev. 116 (2016) 12536-12563.

doi:10.1021/acs.chemrev.6b00369.

[69] S.E. Ahmed, A.M. Martins, G.A. Husseini, The use of ultrasound to release chemotherapeutic drugs from micelles and liposomes., J. Drug Target. 23 (2015) 16-42. doi:10.3109/1061186X.2014.954119.

[70] S. Mitragotri, Healing sound: the use of ultrasound in drug delivery and other therapeutic applications., Nat. Rev. Drug Discov. 4 (2005) 255-60. doi: $10.1038 /$ nrd1662.

[71] C.P. Phenix, M. Togtema, S. Pichardo, I. Zehbe, L. Curiel, High Intensity Focused Ultrasound Technology, its Scope and Applications in Therapy and Drug Delivery, J. Pharm. Pharm. Sci. 17 (2014) 136. doi:10.18433/J3ZP5F.

[72] J. Unga, M. Hashida, Ultrasound induced cancer immunotherapy., Adv. Drug Deliv. Rev. 72 (2014) 144-53. doi:10.1016/j.addr.2014.03.004.

[73] E. Unger, T. Porter, J. Lindner, P. Grayburn, Cardiovascular drug delivery with ultrasound and microbubbles., Adv. Drug Deliv. Rev. 72 (2014) 110-26. doi:10.1016/j.addr.2014.01.012.

[74] M. Aryal, C.D. Arvanitis, P.M. Alexander, N. McDannold, Ultrasound-mediated blood-brain barrier disruption for targeted drug delivery in the central nervous system., Adv. Drug Deliv. Rev. 72 (2014) 94-109. doi:10.1016/j.addr.2014.01.008.

[75] J.J. Rychak, A.L. Klibanov, Nucleic acid delivery with microbubbles and ultrasound., Adv. Drug Deliv. Rev. 72 (2014) 82-93. doi:10.1016/j.addr.2014.01.009.

[76] F. Kiessling, S. Fokong, J. Bzyl, W. Lederle, M. Palmowski, T. Lammers, Recent advances in molecular, multimodal and theranostic ultrasound imaging., Adv. Drug Deliv. Rev. 72 (2014) 15-27. doi:10.1016/j.addr.2013.11.013.

[77] S.R. Sirsi, M.A. Borden, State-of-the-art materials for ultrasound-triggered drug delivery., Adv. Drug Deliv. Rev. 72 (2014) 3-14. doi:10.1016/j.addr.2013.12.010.

[78] S. Ibsen, M. Benchimol, D. Simberg, S. Esener, Ultrasound mediated localized drug delivery., Adv. Exp. Med. Biol. 733 (2012) 145-53. doi:10.1007/978-94-0072555-3 14.

[79] J. Castle, M. Butts, A. Healey, K. Kent, M. Marino, S.B. Feinstein, Ultrasound- 
mediated targeted drug delivery: recent success and remaining challenges., Am. J. Physiol. Heart Circ. Physiol. 304 (2013) H350-7.

doi:10.1152/ajpheart.00265.2012.

[80] J. Owen, Q. Pankhurst, E. Stride, Magnetic targeting and ultrasound mediated drug delivery: benefits, limitations and combination., Int. J. Hyperthermia. 28 (2012) 362-73. doi:10.3109/02656736.2012.668639.

[81] J.T. Sutton, K.J. Haworth, G. Pyne-Geithman, C.K. Holland, Ultrasound-mediated drug delivery for cardiovascular disease, Expert Opin. Drug Deliv. (2013). http://www.tandfonline.com/doi/abs/10.1517/17425247.2013.772578 (accessed June 2, 2016).

[82] A. Azagury, L. Khoury, G. Enden, J. Kost, Ultrasound mediated transdermal drug delivery., Adv. Drug Deliv. Rev. 72 (2014) 127-43.

doi:10.1016/j.addr.2014.01.007.

[83] V. Frenkel, Ultrasound mediated delivery of drugs and genes to solid tumors., Adv. Drug Deliv. Rev. 60 (2008) 1193-208. doi:10.1016/j.addr.2008.03.007.

[84] K.Y. Lee, D.J. Mooney, Hydrogels for Tissue Engineering, Chem. Rev. 101 (2001) 1869-1880. doi:10.1021/cr000108x.

[85] A.S. Hoffman, Hydrogels for biomedical applications, Adv. Drug Deliv. Rev. 64 (2012) 18-23. doi:10.1016/j.addr.2012.09.010.

[86] K.Y. Lee, D.J. Mooney, Alginate: properties and biomedical applications., Prog. Polym. Sci. 37 (2012) 106-126. doi:10.1016/j.progpolymsci.2011.06.003.

[87] J.L. Drury, D.J. Mooney, Hydrogels for tissue engineering: scaffold design variables and applications, Biomaterials. 24 (2003) 4337-4351. doi:10.1016/S0142-9612(03)00340-5.

[88] M.P. Lutolf, J.A. Hubbell, Synthetic biomaterials as instructive extracellular microenvironments for morphogenesis in tissue engineering., Nat. Biotechnol. 23 (2005) 47-55. doi:10.1038/nbt1055.

[89] S. Van Vlierberghe, P. Dubruel, E. Schacht, Biopolymer-based hydrogels as scaffolds for tissue engineering applications: a review., Biomacromolecules. 12 (2011) 1387-408. doi:10.1021/bm200083n.

[90] H.J. van der Linden, S. Herber, W. Olthuis, P. Bergveld, Stimulus-sensitive hydrogels and their applications in chemical (micro)analysis, Analyst. 128 (2003) 325-331. doi:10.1039/b210140h.

[91] N.A. Peppas, J.Z. Hilt, A. Khademhosseini, R. Langer, Hydrogels in Biology and Medicine: From Molecular Principles to Bionanotechnology, Adv. Mater. 18 (2006) 1345-1360. doi:10.1002/adma.200501612.

[92] K. Deligkaris, T.S. Tadele, W. Olthuis, A. van den Berg, Hydrogel-based devices for biomedical applications, Sensors Actuators B Chem. 147 (2010) 765-774. doi:10.1016/j.snb.2010.03.083.

[93] J.L. West, J.A. Hubbell, Photopolymerized hydrogel materials for drug delivery applications, React. Polym. 25 (1995) 139-147. doi:10.1016/09231137(94)00096-N.

[94] T.R. Hoare, D.S. Kohane, Hydrogels in drug delivery: Progress and challenges, Polymer (Guildf). 49 (2008) 1993-2007. doi:10.1016/j.polymer.2008.01.027.

[95] N.A. Peppas, P. Bures, W. Leobandung, H. Ichikawa, Hydrogels in pharmaceutical formulations, Eur. J. Pharm. Biopharm. 50 (2000) 27-46. doi:http://dx.doi.org/10.1016/S0939-6411(00)00090-4.

[96] Y. Qiu, K. Park, Environment-sensitive hydrogels for drug delivery, Adv. Drug 
Deliv. Rev. 53 (2001) 321-339. doi:http://dx.doi.org/10.1016/S0169409X(01)00203-4.

[97] J.K. Oh, R. Drumright, D.J. Siegwart, K. Matyjaszewski, The development of microgels/nanogels for drug delivery applications, Prog. Polym. Sci. 33 (2008) 448-477. doi:10.1016/j.progpolymsci.2008.01.002.

[98] T. Vermonden, R. Censi, W.E. Hennink, Hydrogels for protein delivery., Chem. Rev. 112 (2012) 2853-88. doi:10.1021/cr200157d.

[99] P.Y. Wang, Implantable reservoir for supplemental insulin delivery on demand by external compression, Biomaterials. 10 (1989) 197-201. doi:http://dx.doi.org/10.1016/0142-9612(89)90024-0.

[100] Y. Yang, G. Tang, H. Zhang, Y. Zhao, X. Yuan, Y. Fan, et al., Controlled release of BSA by microsphere-incorporated PLGA scaffolds under cyclic loading, Mater. Sci. Eng. C. 31 (2011) 350-356. doi:http://dx.doi.org/10.1016/j.msec.2010.10.006.

[101] B. Kim, S. Yoo, Y.-J. Kim, J. Park, B. Kang, S. Ham, et al., A Strain-Regulated, Refillable Elastic Patch for Controlled Release, Adv. Mater. Interfaces. (2016) n/an/a. doi:10.1002/admi.201500803.

[102] M.B. Larsen, A.J. Boydston, Successive mechanochemical activation and small molecule release in an elastomeric material., J. Am. Chem. Soc. 136 (2014) 1276-9. doi:10.1021/ja411891x.

[103] K.Y. Lee, M.C. Peters, K.W. Anderson, D.J. Mooney, Controlled growth factor release from synthetic extracellular matrices, Nature. 408 (2000) 998-1000. doi:10.1038/35050141.

[104] K.Y. Lee, M.C. Peters, D.J. Mooney, Controlled Drug Delivery from Polymers by Mechanical Signals, Adv. Mater. 13 (2001) 837-839. doi:10.1002/15214095(200106)13:11<837::AID-ADMA837>3.0.CO;2-D.

[105] F. Hirayama, Cyclodextrin-based controlled drug release system, Adv. Drug Deliv. Rev. 36 (1999) 125-141. doi:10.1016/S0169-409X(98)00058-1.

[106] H. Izawa, K. Kawakami, M. Sumita, Y. Tateyama, J.P. Hill, K. Ariga, $\beta$ Cyclodextrin-crosslinked alginate gel for patient-controlled drug delivery systems: regulation of host-guest interactions with mechanical stimuli, J. Mater. Chem. B. 1 (2013) 2155. doi:10.1039/c3tb00503h.

[107] L. Tan, J. Li, Y. Liu, H. Zhou, Z. Zhang, L. Deng, Synthesis and characterization of $\beta$-cyclodextrin-conjugated alginate hydrogel for controlled release of hydrocortisone acetate in response to mechanical stimulation, J. Bioact. Compat. Polym. 30 (2015) 584-599. doi:10.1177/0883911515590494.

[108] L. Xiao, Z. Tong, Y. Chen, D.J. Pochan, C.R. Sabanayagam, X. Jia, Hyaluronic Acid-Based Hydrogels Containing Covalently Integrated Drug Depots: Implication for Controlling Inflammation in Mechanically Stressed Tissues, Biomacromolecules. 14 (2013) 3808-3819. doi:10.1021/bm4011276.

[109] R. Rajamanickam, S. Baek, K. Gwon, Y. Hwang, K. Shin, G. Tae, Mechanical stimuli responsive and highly elastic biopolymer/nanoparticle hybrid microcapsules for controlled release, J. Mater. Chem. B. (2016). doi:10.1039/C6TB00410E.

[110] Q. Wang, G.R. Gossweiler, S.L. Craig, X. Zhao, Cephalopod-inspired design of electro-mechano-chemically responsive elastomers for on-demand fluorescent patterning, Nat. Commun. 5 (2014) 4899. doi:10.1038/ncomms5899.

[111] D.R. Stabley, C. Jurchenko, S.S. Marshall, K.S. Salaita, Visualizing mechanical tension across membrane receptors with a fluorescent sensor, Nat. Methods. 9 
(2011) 64-67. doi:10.1038/nmeth.1747.

[112] T. Yamada, Y. Hayamizu, Y. Yamamoto, Y. Yomogida, A. Izadi-Najafabadi, D.N. Futaba, et al., A stretchable carbon nanotube strain sensor for human-motion detection, Nat. Nanotechnol. 6 (2011) 296-301. doi:10.1038/nnano.2011.36.

[113] J.A. Rogers, T. Someya, Y. Huang, Materials and mechanics for stretchable electronics., Science. 327 (2010) 1603-7. doi:10.1126/science.1182383.

[114] C. Larson, B. Peele, S. Li, S. Robinson, M. Totaro, L. Beccai, et al., Highly stretchable electroluminescent skin for optical signaling and tactile sensing., Science. 351 (2016) 1071-4. doi:10.1126/science.aac5082.

[115] L. Hu, M. Pasta, F. La Mantia, L. Cui, S. Jeong, H.D. Deshazer, et al., Stretchable, Porous, and Conductive Energy Textiles, Nano Lett. 10 (2010) 708714. doi:10.1021/nl903949m.

[116] S. Wagner, S.P. Lacour, J. Jones, P.I. Hsu, J.C. Sturm, T. Li, et al., Electronic skin: architecture and components, Phys. E Low-Dimensional Syst. Nanostructures. 25 (2004) 326-334. doi:10.1016/j.physe.2004.06.032.

[117] R.F. Riley, C.W. Don, W. Powell, C. Maynard, L.S. Dean, Trends in Coronary Revascularization in the United States From 2001 to 2009: Recent Declines in Percutaneous Coronary Intervention Volumes, Circ. Cardiovasc. Qual. Outcomes. 4 (2011) 193-197. doi:10.1161/CIRCOUTCOMES.110.958744.

[118] P. Calvert, Hydrogels for Soft Machines, Adv. Mater. 21 (2009) 743-756. doi:10.1002/adma.200800534.

[119] J.-Y. Sun, X. Zhao, W.R.K. Illeperuma, O. Chaudhuri, K.H. Oh, D.J. Mooney, et al., Highly stretchable and tough hydrogels., Nature. 489 (2012) 133-6. doi:10.1038/nature11409.

[120] Y. Zhang, Q. Chen, J. Ge, Z. Liu, Controlled display of enzyme activity with a stretchable hydrogel, Chem. Commun. 49 (2013) 9815-9817. doi:10.1039/C3CC45837G.

[121] D.C. Hyun, G.D. Moon, C.J. Park, B.S. Kim, Y. Xia, U. Jeong, Strain-controlled release of molecules from arrayed microcapsules supported on an elastomer substrate, Angew. Chemie. 50 (2011) 724-727. doi:10.1002/anie.201004838.

[122] L. Xiao, J. Zhu, J.D. Londono, D.J. Pochan, X. Jia, Mechano-responsive hydrogels crosslinked by block copolymer micelles, Soft Matter. 8 (2012) 10233. doi:10.1039/c2sm26566d.

[123] J. Di, S. Yao, Y. Ye, Z. Cui, J. Yu, T.K. Ghosh, et al., Stretch-Triggered Drug Delivery from Wearable Elastomer Films Containing Therapeutic Depots, ACS Nano. 9 (2015) 9407-9415. doi:10.1021/acsnano.5b03975.

[124] D.M. Arm, A.F. Tencer, Effects of cyclical mechanical stress on the controlled release of proteins from a biodegradable polymer implant, J. Biomed. Mater. Res. 35 (1997) 433-441. doi:10.1002/(SICI)1097-4636(19970615)35:4<433::AIDJBM3>3.0.CO;2-I.

[125] Z. Tang, Y. Wang, P. Podsiadlo, N.A. Kotov, Biomedical Applications of Layer-byLayer Assembly: From Biomimetics to Tissue Engineering, Adv. Mater. 18 (2006) 3203-3224. doi:10.1002/adma.200600113.

[126] D. Volodkin, A. Skirtach, H. Möhwald, Bioactive Surfaces, Springer, Berlin, Heidelberg, 2011. doi:10.1007/12_2010_79.

[127] P.T. Hammond, Form and Function in Multilayer Assembly: New Applications at the Nanoscale, Adv. Mater. 16 (2004) 1271-1293. doi:10.1002/adma.200400760.

[128] B.-S. Kim, J.-W. Choi, Polyelectrolyte multilayer microcapsules: Self-assembly 
and toward biomedical applications, Biotechnol. Bioprocess Eng. 12 (2007) 323332. doi:10.1007/BF02931052.

[129] M.A. Stuart, W.T. Huck, J. Genzer, M. Muller, C. Ober, M. Stamm, et al., Emerging applications of stimuli-responsive polymer materials, Nat. Mater. 9 (2010) 101-113. doi:10.1038/nmat2614.

[130] D. Mertz, J. Hemmerlé, J. Mutterer, S. Ollivier, J.-C. Voegel, P. Schaaf, et al., Mechanically responding nanovalves based on polyelectrolyte multilayers., Nano Lett. 7 (2007) 657-62. doi:10.1021/nl062657+.

[131] D. Mertz, C. Vogt, J. Hemmerlé, J. Mutterer, V. Ball, J.-C. Voegel, et al., Mechanotransductive surfaces for reversible biocatalysis activation, Nat. Mater. 8 (2009) 731-735. doi:10.1038/nmat2504.

[132] C. Vogt, D. Mertz, K. Benmlih, J. Hemmerlé, J.-C. Voegel, P. Schaaf, et al., Layer-by-Layer Enzymatic Platform for Stretched-Induced Reactive Release, ACS Macro Lett. 1 (2012) 797-801. doi:10.1021/mz3000896.

[133] J. Barthes, D. Mertz, C. Bach, M.-H. Metz-Boutigue, B. Senger, J.-C. Voegel, et al., Stretch-Induced Biodegradation of Polyelectrolyte Multilayer Films for Drug Release, Langmuir. 28 (2012) 13550-13554. doi:10.1021/la302550q.

[134] E.J. Falde, S.T. Yohe, Y.L. Colson, M.W. Grinstaff, Superhydrophobic materials for biomedical applications, Biomaterials. 104 (2016) 87-103. doi:10.1016/j.biomaterials.2016.06.050.

[135] W. Choi, A. Tuteja, S. Chhatre, J.M. Mabry, R.E. Cohen, G.H. McKinley, Fabrics with Tunable Oleophobicity, Adv. Mater. 21 (2009) 2190-2195. doi:10.1002/adma.200802502.

[136] A. Tuteja, W. Choi, M. Ma, J.M. Mabry, S.A. Mazzella, G.C. Rutledge, et al., Designing superoleophobic surfaces., Science. 318 (2007) 1618-22. doi:10.1126/science.1148326.

[137] J. Zhang, X. Lu, W. Huang, Y. Han, Reversible Superhydrophobicity to Superhydrophilicity Transition by Extending and Unloading an Elastic Polyamide Film, Macromol. Rapid Commun. 26 (2005) 477-480. doi:10.1002/marc.200400512.

[138] C.R. Crick, I.P. Parkin, Preparation and Characterisation of Super-Hydrophobic Surfaces, Chem. - A Eur. J. 16 (2010) 3568-3588. doi:10.1002/chem.200903335.

[139] X. Zhang, F. Shi, J. Niu, Y. Jiang, Z. Wang, Superhydrophobic surfaces: from structural control to functional application, J. Mater. Chem. 18 (2008) 621-633. doi:10.1039/B711226B.

[140] X. Huang, Y. Sun, S. Soh, Stimuli-Responsive Surfaces for Tunable and Reversible Control of Wettability., Adv. Mater. 27 (2015) 4062-8. doi:10.1002/adma.201501578.

[141] J. Wang, J.A. Kaplan, Y.L. Colson, M.W. Grinstaff, Stretch-Induced Drug Delivery from Superhydrophobic Polymer Composites: Use of Crack Propagation Failure Modes for Controlling Release Rates, Angew. Chemie. 128 (2016) 2846-2850. doi:10.1002/ange.201511052.

[142] M. Guvendiren, H.D. Lu, J.A. Burdick, Shear-thinning hydrogels for biomedical applications, Soft Matter. 8 (2012) 260-272. doi:10.1039/C1SM06513K.

[143] T. Saxer, A. Zumbuehl, B. Müller, The use of shear stress for targeted drug delivery., Cardiovasc. Res. 99 (2013) 328-33. doi:10.1093/cvr/cvt102.

[144] N. Korin, M.J. Gounis, A.K. Wakhloo, D.E. Ingber, Targeted Drug Delivery to Flow-Obstructed Blood Vessels Using Mechanically Activated Nanotherapeutics., 
JAMA Neurol. 72 (2014) 1-4. doi:10.1001/jamaneurol.2014.2886.

[145] T. Natsume, M. Yoshimoto, Mechanosensitive Liposomes as Artificial Chaperones for Shear- Driven Acceleration of Enzyme-Catalyzed Reaction, ACS Appl. Mater. Interfaces. 6 (2014) 3671-3679. doi:10.1021/am405992t.

[146] M.N. Holme, I. a. Fedotenko, D. Abegg, J. Althaus, L. Babel, F. Favarger, et al., Shear-stress sensitive lenticular vesicles for targeted drug delivery, Nat. Nanotechnol. 7 (2012) 536-543. doi:10.1038/nnano.2012.84.

[147] N. Korin, M. Kanapathipillai, B.D. Matthews, M. Crescente, A. Brill, T. Mammoto, et al., Shear-Activated Nanotherapeutics for Drug Targeting to Obstructed Blood Vessels, Science (80-. ). 337 (2012) 738-742. doi:10.1126/science.1217815.

[148] M.G. Marosfoi, N. Korin, M.J. Gounis, O. Uzun, S. Vedantham, E.T. Langan, et al., Shear-Activated Nanoparticle Aggregates Combined With Temporary Endovascular Bypass to Treat Large Vessel Occlusion, Stroke. 46 (2015).

[149] C. Chen, S. Li, K. Liu, G. Ma, X. Yan, Co-Assembly of Heparin and Polypeptide Hybrid Nanoparticles for Biomimetic Delivery and Anti-Thrombus Therapy, Small. 12 (2016) 4719-4725. doi:10.1002/smll.201600328.

[150] A.C. Anselmo, C.L. Modery-Pawlowski, S. Menegatti, S. Kumar, D.R. Vogus, L.L. Tian, et al., Platelet-like Nanoparticles (PLNs): Engineering Shape, Flexibility and Surface Chemistry of Nanocarriers to Target Vascular Injuries., ACS Nano. 8 (2014) 11243-11253. doi:10.1021/nn503732m.

[151] L. Mellal, K. Belharet, D. Folio, A. Ferreira, Optimal structure of particles-based superparamagnetic microrobots: application to MRI guided targeted drug therapy, J. Nanoparticle Res. 17 (2015) 64. doi:10.1007/s11051-014-2733-3.

[152] J.A. Kaplan, P. Barthélémy, M.W. Grinstaff, Self-assembled nanofiber hydrogels for mechanoresponsive therapeutic anti-TNFa antibody delivery., Chem. Commun. (Camb). 52 (2016) 5860-3. doi:10.1039/c6cc02221a.

[153] L. Xiao, J. Zhu, D.J. Londono, D.J. Pochan, X. Jia, Mechano-Responsive Hydrogels Crosslinked by Block Copolymer Micelles., Soft Matter. 8 (2012) 10233-10237. doi:10.1039/C2SM26566D. 


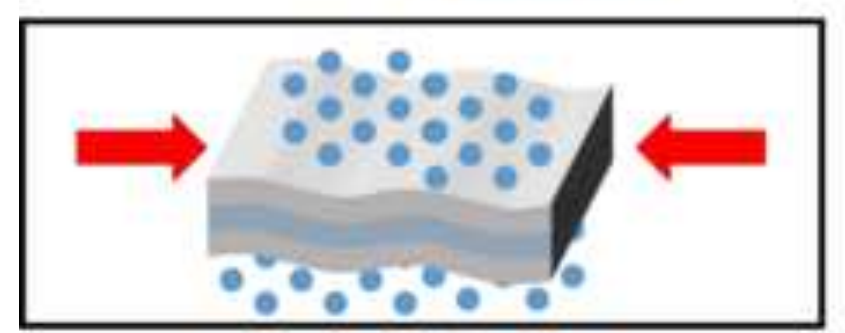

Compression

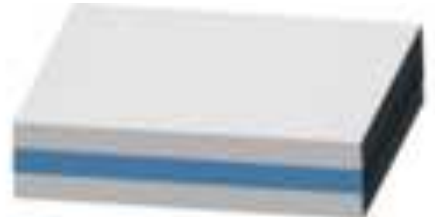

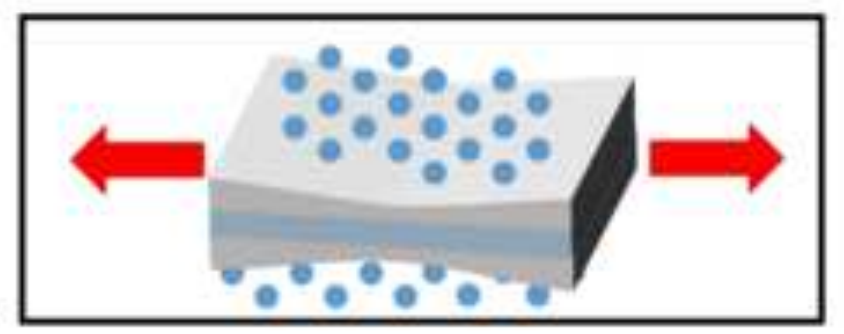

Tension

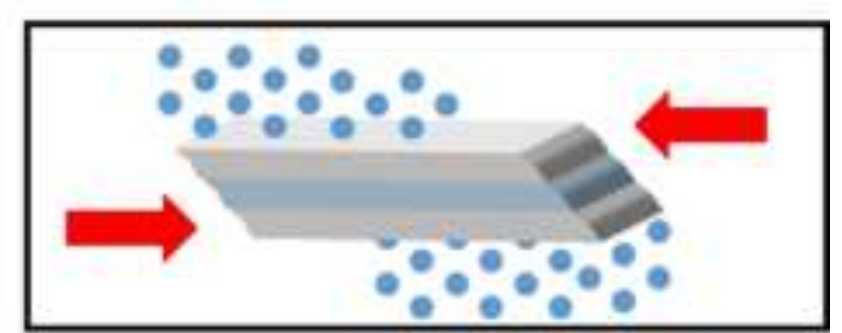

Shear 